\title{
Operators Between Subspaces and Quotients of $L^{1}$
}

G. Godefroy, N. J. Kalton \& D. Li

ABSTRACT. We provide an unified approach of results of L. Dor on the complementation of the range, and of D. Alspach on the nearness from isometries, of small intoisomorphisms of $L^{1}$. We introduce the notion of small subspace of $L^{1}$, and show lifting theorems for operators between quotients of $L^{1}$ by small subspaces. We construct a subspace of $L^{1}$ which shows that extension of isometries from subspaces of $L^{1}$ to the whole space are no longer true for isomorphisms, and that nearly isometric isomorphisms from subspaces of $L^{1}$ into $L^{1}$ need not be near from any isometry.

I. Introduction . . . . . . . . . . . . . . . . . . 245

II. Projections and small into-isomorphisms of $L^{1} \ldots \ldots 248$

III. Small subspaces of $L^{1} \ldots \ldots$. . . . . . . . . . . . . . . . 260

IV. Lifting of operators between quotients by small subspaces of $L^{1}$. 266

V. Construction of a peculiar subspace of $L^{1} \ldots \ldots$. . . . . . 274

I. Introduction. The Banach space $L^{1}$ is of fundamental importance for Fourier analysis, probability theory, and many other fields of pure and applied analysis. However, its Banach space structure is not yet fully understood. In particular, the study of operators from $L^{1}$ to $L^{1}$ lead to simply stated but apparently very hard problems: for instance, it is not known whether a complemented subspace of $L^{1}$ is necessarily isomorphic to $L^{1}$ or $\ell^{1}$ (see [35]). A major tool for handling operators on $L^{1}(\Omega)$ is their representation through a "matrix" whose "rows" are indexed by the points of $\Omega$ and consist of measures on $\Omega$ ([18]; see also [9]). This representation 
will play a major role in the present work, together with duality arguments, which will allow us to use methods and results from the isometric theory of Banach spaces. We will focus on several natural aspects of operator theory on $L^{1}$ : perturbation of operators which are close to isometries, lifting of operators between quotient spaces, extensions of operators defined on linear subspaces. Such questions have of course been considered before and important theorems have been obtained (see e.g. [1], [7], [8], [15], [19], [21], [32], and references therein). Our work provides on one hand alternative simpler proofs to some known theorems, which are subsequently improved, and on the other hand new statements. We now turn to a detailed description of our results.

Section II begins with a simple inequality, whose proof relies on Gaussian randomization, which provides a control of the atomic part of normincreasing operators (Lemma II.1). This inequality leads to a unified approach to Dor's complementation theorem (Theorem II.3) and to Alspach's perturbation theorem (Theorem II.7). These two results follow from a Hahn-Banach argument applied to the atomic part of the relevant operator, considered as taking values in a vector-valued $L^{1}$. Let us mention that our approach provides a quantitative improvement of Alspach's theorem, but do not improves the constant $\sqrt{2}$ in the statement of Dor's result. The new notion of "small subspace" is introduced in Section III (Definition III.1). Roughly speaking, a "small subspace" is a subspace of $L^{1}$ which is nowhere "locally equal" to $L^{1}$. Operators which have a non trivial "diagonal" in their "matrix" representation are denoted strong Enflo operators (Definition III.3). The link between these two notions is that operators which take their values in a small subspace are not strong Enflo; in fact, more is true (Proposition III.6). Small subspaces are studied within the class of translation invariant subspaces; it is also shown that a closed direct sum of two small subspaces is small (Proposition III.9). Smallness of spaces is a crucial concept in Section IV, which is devoted to the lifting of operators between quotient spaces: the main result of this section is that, if $X$ and $Y$ are small subspaces, and the quotient spaces $L^{1} / X$ and $L^{1} / Y$ are close to each other in Banach-Mazur distance, then there is an isomorphism of $L^{1}$ which maps $X$ close to $Y$ with respect to the Hausdorff distance (Theorem IV.3). When one of the spaces $X$ or $Y$ has the lifting property (e.g. if one of these spaces is complemented in its bidual), it follows that there is an invertible operator of $L^{1}$ which maps $X$ onto $Y$ (Corollary IV.8). This result can be seen as a substitute in $L^{1}$ of a theorem of Lindenstrauss and Rosenthal on $\ell^{1}$ ([24]; see [25], Th.2.f.8). Note, however, that the quantitative behaviour of the liftings is actually better in the $L^{1}$ case (see Remark IV.9). Finally, 
Section $V$ provides a counterexample, showing the "sensibility over $\varepsilon$ " of the positive results obtained so far about isometries: there is a subspace $X$ of $L^{1}$ such that, although isometries from this space into $L^{1}$ extend to isometries of the whole space $L^{1}$, there are linear maps $T$ from $X$ into $L^{1}$, which are arbitrarily close to isometries, and such that no small perturbation of $T$ is actually an isometry from $X$ into $L^{1}$; in particular, these "near isometries" do not extend to near isometries defined on $L^{1}$ (Theorem V.1). This space $X$ is arbitrarily close in the Banach-Mazur sense to weak-star closed subspaces of $\ell^{1}$, though it is far in the Hausdorff distance of any subspace of $L^{1}$ spanned by disjoint functions; hence, although it has a strong " $\ell$ " " behaviour (for instance, its unit ball is compact locally convex in measure, see [12]), it cannot be decomposed into essentially disjoint finite dimensional parts (compare with [7], [33]). The construction of $X$ makes a crucial use of $p$-stable random variables with $p$ close to 1 .

Our notation is classical. All the Banach spaces we consider are indifferently real or complex, except in Section V, where we work only in real case. All the measure spaces are separable and purely non-atomic, and the measures are (unless other stated) probability measures; hence the corresponding $L^{1}$-spaces are isometric to $L^{1}(0,1)$, but for convenience we use sometimes a more general probability space. When writing $L^{1}([0,1], m)$, we understand that $m$ is a Lebesgue measure. The following representation of operators on $L^{1}$ ([18], Theorem 3.1) will be very useful: for every operator $T: L^{1} \rightarrow L^{1}$ there are measures $\mu_{x}$, with $x \mapsto \mu_{x}$ weak $^{*}$-measurable, such that, for almost every $x$,

$$
T f(x)=\int_{0}^{1} f(s) d \mu_{x}(s)=\sum_{n=1}^{+\infty} a_{n}(x) f\left(\sigma_{n}(x)\right)+\int_{0}^{1} f(s) d v_{x}(s),
$$

where $\sum_{n=1}^{+\infty} a_{n}(x) \delta_{\sigma_{n}(x)}$ and $v_{x}$ are the atomic and continuous part of $\mu_{x}$.

Section III and IV are essentially independent of Section II.

Acknowledgement. This work was initiated while the first author was Visiting Professor at the University of Missouri-Columbia during the academic year 1996-1997, and the third author was visiting this University in the fall of 1996. It is their pleasure to thank the Department of mathematics of U.M.C. for its hospitality and support, and all persons and institutions which made these stays possible.

The second author was supported in part by NSF grant DMS-9870027. 
II. Projections and small into-isomorphisms of $L^{1}$. In this section, we provide a unified approach to results of D. Alspach and L. Dor on isomorphisms from $L^{1}$ into $L^{1}$ with small bounds. A short proof of the special case of convolutors, which points towards the main lemma, will be given at the end of the section (see Remark II.8). The key result of this approach is the following lemma:

Lemma II.1. Let $T: L^{1}([0,1], m) \rightarrow L^{1}([0,1], m)$, and write

$$
T f(s)=\sum_{n=1}^{\infty} a_{n}(s) f\left(\sigma_{n}(s)\right)+\int_{0}^{1} f(t) d v_{s}(t)
$$

Suppose that $\|T f\|_{1} \geq\|f\|_{1}$ for all $f \in L^{1}$. Then, for all $f \in L^{1}$,

$$
\|f\|_{1} \leq\left\|\left(\sum_{k=1}^{\infty}\left|a_{k}(s)\right|^{2}\left|f\left(\sigma_{k}(s)\right)\right|^{2}\right)^{1 / 2}\right\|_{1} .
$$

Proof. Let $\left(\gamma_{k}\right)$ be a sequence of i.i.d. Gaussian (real or complex) random variables on a probability space $(\Omega, \mathbb{P})$, normalized in $L^{1}$. For any $l>0$, we let

$$
D_{k}^{l}=\left[\frac{k-1}{2^{l}}, \frac{k}{2^{l}}\left[, \quad 1 \leq k \leq 2^{l},\right.\right.
$$

be the dyadic intervals of level $l$.

Fact. Let $v$ be a diffuse measure on $[0,1]$. Defining

$$
\varphi_{l}=\sum_{k=1}^{2^{l}}\left|\nu\left(D_{k}^{l}\right)\right|^{2},
$$

one has $\lim _{l} \varphi_{l}=0$.

Indeed, $\varphi_{l} \leq\|v\|_{1} \cdot \max _{k}\left(\left|\nu\left(D_{k}^{l}\right)\right|\right)$, since $\left(\sum\left|\lambda_{k}\right|^{2}\right) \leq\left(\sum\left|\lambda_{k}\right|\right) \cdot\left(\max \left|\lambda_{k}\right|\right)$ for every sequence $\left(\lambda_{k}\right)$ of scalars. Since $v$ is diffuse, this $\max _{k}$ tends to 0 when $l$ goes to infinity, and the conclusion follows.

Let now $\rho$ be an arbitrary measure on $[0,1]$. Let us write

$$
\rho=\sum_{n=1}^{\infty} a_{n} \delta_{x_{n}}+v=\alpha+v
$$


where $\alpha$ is atomic and $v$ is diffuse. For any $l$ and $N=2^{l}$, one has

$$
\sum_{k=1}^{N}\left|\rho\left(D_{k}^{l}\right)\right|^{2}=\sum_{k=1}^{N}\left|\alpha\left(D_{k}^{l}\right)+\nu\left(D_{k}^{l}\right)\right|^{2},
$$

and from the Fact, it follows easily that

$$
\lim _{N} \sum_{k=1}^{N}\left|\rho\left(D_{k}^{l}\right)\right|^{2}=\sum_{n=1}^{\infty}\left|a_{n}\right|^{2} .
$$

We now denote $D_{k}^{l}=D_{k}$, and for a given $N=2^{l}$, any $\omega \in \Omega$, and any $f \in L^{1}$, we define

$$
f_{\omega}=\sum_{k=1}^{N} \gamma_{k}(\omega) \mathbf{1}_{D_{k}} f .
$$

With this notation, one has

$$
\begin{aligned}
\int_{\Omega}\left\|f_{\omega}\right\|_{1} d \mathbb{P}(\omega) & =\iint_{[0,1] \times \Omega}\left|f_{\omega}(t)\right| d t d \mathbb{P}(\omega) \\
& =\iint_{[0,1] \times \Omega}\left|\sum_{k=1}^{N} \gamma_{k}(\omega) \mathbf{1}_{D_{k}}(t) f(t)\right| d t d \mathbb{P}(\omega) \\
& =\int_{\Omega}\left(\sum_{k=1}^{N}\left|\gamma_{k}(\omega)\right| \int_{D_{k}}|f(t)| d t\right) d \mathbb{P}(\omega)=\|f\|_{1},
\end{aligned}
$$

and it follows from the assumption on $T$ that, for any $f$, one has

$$
\|f\|_{1} \leq \int_{\Omega}\left\|T\left(f_{\omega}\right)\right\|_{1} d \mathbb{P}(\omega)
$$

We now use the representation (R) of $T$

(R) $T f(s)=\int_{0}^{1} f(t) d \mu_{s}(t)=\sum_{n=1}^{\infty} a_{n}(s) f\left(\sigma_{n}(s)\right)+\int_{0}^{1} f(t) d v_{s}(t)$,

and for any given $f \in L^{1}$ we define, for all $s$, a measure $\rho_{s}$ by $d \rho_{s}=f \cdot d \mu_{s}$. 
With this notation, one has

$$
T\left(f_{\omega}\right)(s)=\sum_{k=1}^{N} \gamma_{k}(\omega) \rho_{s}\left(D_{k}\right)
$$

and thus, by Fubini's Theorem,

$$
\begin{aligned}
\int_{\Omega}\left\|T\left(f_{\omega}\right)\right\|_{1} d \mathbb{P}(\omega) & =\int_{0}^{1} \int_{\Omega}\left|\sum_{k=1}^{N} \gamma_{k}(\omega) \rho_{s}\left(D_{k}\right)\right| d \mathbb{P}(\omega) d s \\
& =\int_{0}^{1}\left(\sum_{k=1}^{N}\left|\rho_{s}\left(D_{k}\right)\right|^{2}\right)^{1 / 2} d s .
\end{aligned}
$$

It follows that

$$
\|f\|_{1} \leq \int_{0}^{1}\left(\sum_{k=1}^{N}\left|\rho_{s}\left(D_{k}\right)\right|^{2}\right)^{1 / 2} d s .
$$

On the other hand, by applying (1) to $\rho=\rho_{s}$, we have for almost every $s$ :

$$
\lim _{N}\left(\sum_{k=1}^{N}\left|\rho\left(D_{k}\right)\right|^{2}\right)^{1 / 2}=\left(\sum_{n=1}^{\infty}\left|a_{n}(s) f\left(\sigma_{n}(s)\right)\right|^{2}\right)^{1 / 2} .
$$

Since (R) implies that

$$
\left\|\rho_{s}\right\| \leq|T|(|f|)(s),
$$

we may apply the dominated convergence theorem, and it follows then that

$$
\|f\|_{1} \leq\left\|\left(\sum_{k=1}^{\infty}\left|a_{k}(s)\right|^{2}\left|f\left(\sigma_{k}(s)\right)\right|^{2}\right)^{1 / 2}\right\|_{1},
$$

which is Lemma 1.

Lemma II.2. Let $T: L^{1}([0,1], m) \rightarrow L^{1}([0,1], m)$ and $\alpha \geq 1$ be such that $\alpha\|f\|_{1} \geq\|T f\|_{1} \geq\|f\|_{1}$ for all $f \in L^{1}$. Write

$$
T f(s)=\sum_{n=1}^{\infty} a_{n}(s) f\left(\sigma_{n}(s)\right)+\int_{0}^{1} f(t) d v_{s}(t) .
$$


Then, for all $f \in L^{1}$,

$(* *)$

$$
\begin{aligned}
& \left\|\sum_{n=1}^{\infty}\left|a_{n}(s)\right|\left|f\left(\sigma_{n}(s)\right)\right|\right\|_{1} \leq \alpha\|f\|_{1}, \\
& \alpha^{-1}\|f\|_{1} \leq\left\|\max _{n}\left|a_{n}(s)\right|\left|f\left(\sigma_{n}(s)\right)\right|\right\|_{1} .
\end{aligned}
$$

Proof. It follows e.g. from (R) that $\||T|\|=\|T\|$, hence $\||T|(f)\|_{1} \leq$ $\alpha\|f\|_{1}$, which shows $(*)$. For $(* *)$ we observe that, for any $s \in[0,1]$,

$$
\begin{aligned}
& \left(\sum_{k=1}^{\infty}\left|a_{k}(s)\right|^{2}\left|f\left(\sigma_{k}(s)\right)\right|^{2}\right)^{1 / 2} \\
& \quad \leq\left(\sum_{k=1}^{\infty}\left|a_{k}(s)\right|\left|f\left(\sigma_{k}(s)\right)\right|\right)^{1 / 2}\left(\max _{k}\left|a_{k}(s)\right|\left|f\left(\sigma_{k}(s)\right)\right|\right)^{1 / 2},
\end{aligned}
$$

hence by the Cauchy-Schwarz inequality

$$
\begin{aligned}
& \left\|\left(\sum_{k=1}^{\infty}\left|a_{k}(s)\right|^{2}\left|f\left(\sigma_{k}(s)\right)\right|^{2}\right)^{1 / 2}\right\|_{1} \\
& \quad \leq\left\|\left(\sum_{k=1}^{\infty}\left|a_{k}(s)\right|\left|f\left(\sigma_{k}(s)\right)\right|\right)\right\|_{1}^{1 / 2} \cdot\left\|\max _{k}\left|a_{k}(s)\right|\left|f\left(\sigma_{k}(s)\right)\right|\right\|_{1}^{1 / 2} .
\end{aligned}
$$

It follows now from $(*)$ and Lemma 1 that

$$
\|f\|_{1} \leq \sqrt{\alpha}\|f\|_{1}^{1 / 2}\left\|\max _{k}\left|a_{k}(s)\right|\left|f\left(\sigma_{k}(s)\right)\right|\right\|_{1}^{1 / 2},
$$

which shows $(* *)$.

Lemma II.2 allows us to show the following theorem due to L. Dor ([7], Corollary 3.3) through a Hahn-Banach argument, distinct however from the original argument ([7]). Note that we do not have the better constant 1.6 obtained by L. Dor in the real case.

Theorem II.3. Let $T: L^{1} \rightarrow L^{1}$ be an isomorphism into $L^{1}$ such that $\|T\|$. $\left\|T^{-1}\right\|<\sqrt{2}$, with $T^{-1}: T\left(L^{1}\right) \rightarrow L^{1}$. Then $T\left(L^{1}\right)$ is a complemented subspace of $L^{1}$. 
Proof. We may and do assume that $T$ satisfies the assumptions of Lemma II.2, with $\alpha<\sqrt{2}$. Define $\Psi: L^{1} \rightarrow L^{1}\left(c_{0}\right)$ by

$$
\Psi(f)=\left(\left|a_{n}(s)\right| f\left(\sigma_{n}(s)\right)\right)_{n \geq 1} .
$$

Note that, by $(*), \Psi$ actually takes its values in $L^{1}\left(\ell_{1}\right)$. By $(* *)$, we have

$$
\|\Psi(f)\|_{L^{1}\left(c_{0}\right)} \geq \alpha^{-1}\|f\|_{1} \quad \text { for all } f \in L^{1} .
$$

Hence, there exists $F \in L^{1}\left(c_{0}\right)^{*}=L^{\infty}\left(\ell_{1}\right)$, with $F \geq 0,\|F\| \leq \alpha$, such that

$$
\int_{0}^{1} f(s) d s=F[\Psi(f)] \quad \text { for all } f \in L^{1} .
$$

That means there exists a sequence $\left(b_{n}\right)_{n \geq 1}$ of positive functions such that

$$
\left\|\sum_{n=1}^{\infty} b_{n}(s)\right\|_{\infty} \leq \alpha
$$

and

$$
\int_{0}^{1} f(s) d s=\int_{0}^{1} \sum_{n=1}^{\infty} b_{n}(s)\left|a_{n}(s)\right| f\left(\sigma_{n}(s)\right) d s \quad \text { for all } f \in L^{1}
$$

Choose disjoint measurable subsets $E_{n}, n \geq 1$, of $[0,1] \times[0,1]$ such that

$$
m\left(\left\{x \mid(s, x) \in E_{n}\right\}\right)=\frac{b_{n}(s)}{\alpha} \quad \text { for all } s \in[0,1], n \geq 1 .
$$

We define $U: L^{1}([0,1]) \rightarrow L^{1}\left([0,1]^{2}\right)$ by

$$
U f(s, x)=\sum_{n=1}^{\infty} a_{n}(s) f\left(\sigma_{n}(s)\right) \mathbf{1}_{E_{n}}(s, x)
$$

For any $f \in L^{1}$, one has

$$
\|U f\|_{1}=\int_{0}^{1} \sum_{n=1}^{\infty} \frac{b_{n}(s)}{\alpha}\left|a_{n}(s)\right|\left|f\left(\sigma_{n}(s)\right)\right| d s=\frac{1}{\alpha}\|f\|_{1} .
$$


Hence $U\left(L^{1}\right)$ is isometric to $L^{1}$, and it follows (see [22], Chap. 6, Section 17 , Theorem 3$)$ that $U\left(L^{1}\right)$ is 1 -complemented in $L^{1}\left([0,1]^{2}\right)$.

Define $\bar{T}: L^{1}([0,1]) \rightarrow L^{1}\left([0,1]^{2}\right)$ by

$$
\bar{T} f(s, x)=T f(s)=\sum_{n=1}^{\infty} a_{n}(s) f\left(\sigma_{n}(s)\right)+\int_{0}^{1} f(u) d v_{s}(u) .
$$

If we denote

$$
\begin{aligned}
& U f(s, x)=\int_{0}^{1} f(u) d \mu_{(s, x)}^{U}(u), \\
& \bar{T} f(s, x)=\int_{0}^{1} f(u) d \mu_{(s, x)}^{\bar{T}}(u),
\end{aligned}
$$

then clearly

$$
\mu_{(s, x)}^{U}=a_{k}(s) \delta_{\sigma_{k}(s)}
$$

for the unique $k \geq 1$ such that $(s, x) \in E_{k}$, and

$$
\mu_{(s, x)}^{T}=\sum_{n=1}^{\infty} a_{n}(s) \delta_{\sigma_{n}(s)}+v_{s},
$$

for all $(s, x)$. Hence, we have

$$
\mu_{(s, x)}^{\bar{T}-U}=\sum_{n \neq k} a_{n}(s) \delta_{\sigma_{n}(s)}+v_{s}
$$

and it easily follows that

$$
|\bar{T}|-|U|=|\bar{T}-U| \geq 0 .
$$

Note that for all $f \in L^{1}$ :

$$
\||U|(f)\|_{1}=\|U f\|_{1}=\frac{1}{\alpha}\|f\|_{1} .
$$

On the other hand,

$$
\||\bar{T}|(f)\|_{1}=\||T|(f)\|_{1} \leq \alpha\|f\|_{1} .
$$


Hence, for any $f \geq 0$,

$$
\begin{aligned}
\||\bar{T}-U|(f)\|_{1} & =\||\bar{T}|(f)-|U|(f)\|_{1}=\||\bar{T}|(f)\|_{1}-\||U|(f)\|_{1} \\
& \leq\left(\alpha-\alpha^{-1}\right)\|f\|_{1}=\left(\alpha^{2}-1\right)\|U f\|_{1} .
\end{aligned}
$$

Observe now that $U$ maps disjoint functions to disjoint functions, hence if $f=f^{+}-f^{-}$

$$
\|U f\|_{1}=\left\|U f^{+}\right\|_{1}+\left\|U f^{-}\right\|_{1},
$$

and thus for any $g \in L^{1}$ :

$$
\|(\bar{T}-U) g\|_{1} \leq\left(\alpha^{2}-1\right)\|U g\|_{1}=\beta\|U g\|_{1} .
$$

Since $\alpha<\sqrt{2}$, we have $\beta=\alpha^{2}-1<1$. Let $\Pi: L^{1}\left([0,1]^{2}\right) \rightarrow U\left(L^{1}\right)$ be a projection with $\|\Pi\|=1$. It follows from (2) that $\Pi_{\mid \bar{T}\left(L^{1}\right)}$ is an isomorphism from $\bar{T}\left(L^{1}\right)$ onto $U\left(L^{1}\right)$.

Let $S=\Pi^{-1}: U\left(L^{1}\right) \rightarrow \bar{T}\left(L^{1}\right)$. It is easily seen that $S \Pi$ defines a projection from $L^{1}\left([0,1]^{2}\right)$ onto $\bar{T}\left(L^{1}\right)$. Restricting $S \Pi$ to the functions which are independent of $x$, gives a projection from $L^{1}$ to $T\left(L^{1}\right)$.

Our goal is now to provide a similar approach to Alspach's result on near isometries ([1]). We first prove:

Theorem II.4. Let $T: L^{1} \rightarrow L^{1}$ be such that

$$
\alpha\|f\|_{1} \geq\|T f\|_{1} \geq\|f\|_{1} \quad \text { for all } f \in L^{1} .
$$

Then for each $\varepsilon>0$, there exists an operator $S_{\varepsilon}: L^{1} \rightarrow L^{1}$ of the form

$$
S_{\varepsilon} f(s)=\sum_{k=1}^{N} c_{k}(s) f\left(\sigma_{k}(s)\right),
$$

and such that

$$
\left\|T-S_{\varepsilon}\right\| \leq(\alpha-1)+\varepsilon .
$$

Proof. We consider again

$$
\Psi(f)=\left(\left|a_{n}(s)\right| f\left(\sigma_{n}(s)\right)\right)_{n \geq 1},
$$

but this time as an operator from $L^{1}$ to $L^{1}\left(\ell_{2}\right)$. By Lemma 1 , we have for all $f$

$$
\|f\|_{1} \leq\|\Psi(f)\|_{L^{1}\left(\ell_{2}\right)} .
$$


Since $L^{1}\left(\ell_{2}\right)^{*}=L^{\infty}\left(\ell_{2}\right)$, there is $\left(b_{n}\right)_{n \geq 1}$ with $b_{n} \geq 0$ such that

$$
\left\{\begin{array}{l}
\left\|\left(\sum b_{n}^{2}\right)^{1 / 2}\right\|_{\infty} \leq 1, \\
\int_{0}^{1} \sum b_{n}(s)\left|a_{n}(s)\right| f\left(\sigma_{n}(s)\right) d s=\int_{0}^{1} f d s,
\end{array}\right.
$$

for all $f$. We may and do redefine the $a_{n}, b_{n}, \sigma_{n}$ 's in such a way that $b_{1} \geq b_{2} \geq \cdots$; hence $0 \leq b_{n}(s) \leq 1 / \sqrt{n}$ for all $s$ and $n$. Then

$$
\begin{aligned}
& \int_{0}^{1}\left|\sum_{n=N+1}^{\infty} b_{n}(s) a_{n}(s) f\left(\sigma_{n}(s)\right)\right| d s \\
& \quad \leq \frac{1}{\sqrt{N}} \int_{0}^{1} \sum_{n=N+1}^{\infty}\left|a_{n}(s)\right|\left|f\left(\sigma_{n}(s)\right)\right| d s \leq \frac{1}{\sqrt{N}}\|T\| \cdot\|f\|_{1},
\end{aligned}
$$

thus

$$
\int_{0}^{1} \sum_{n=1}^{N} b_{n}(s)\left|a_{n}(s)\right|\left|f\left(\sigma_{n}(s)\right)\right| d s \geq\|f\|_{1}-\frac{1}{\sqrt{N}}\|T\| \cdot\|f\|_{1} .
$$

We let

$$
S_{N} f(s)=\sum_{n=1}^{N} a_{n}(s) b_{n}(s) f\left(\sigma_{n}(s)\right)
$$

For any $s$

$$
\mu_{s}^{T-S_{N}}=\sum_{n=1}^{N} a_{n}(s)\left(1-b_{n}(s)\right) \delta_{\sigma_{n}(s)}+v_{s}+\sum_{n=N+1}^{\infty} a_{n}(s) \delta_{\sigma_{n}(s)},
$$

therefore $\left|T-S_{N}\right|=|T|-\left|S_{N}\right|$, and for any $f \geq 0$, one has

$$
\left\|\left|T-S_{N}\right|(f)\right\|_{1}=\left\||T|(f)-\left|S_{N}\right|(f)\right\|_{1}=\||T|(f)\|_{1}-\left\|\left|S_{N}\right|(f)\right\|_{1} .
$$

Since

$\||T|(f)\|_{1} \leq \alpha\|f\|_{1} \quad$ and $\quad\left\|\left|S_{N}\right|(f)\right\|_{1} \geq\|f\|_{1}-\frac{1}{\sqrt{N}}\|T\| \cdot\|f\|_{1}$, 
one has, for all $f \geq 0$,

$$
\left\|\left|T-S_{N}\right|(f)\right\|_{1} \leq(\alpha-1)\|f\|_{1}+\frac{1}{\sqrt{N}}\|T\| \cdot\|f\|_{1},
$$

and thus

$$
\left\|T-S_{N}\right\|=\left\|\left|T-S_{N}\right|\right\| \leq(\alpha-1)+\frac{\|T\|}{\sqrt{N}} .
$$

The conclusion follows, since $N$ is arbitrary.

Proposition II.5. Let $S: L^{1} \rightarrow L^{1}$ be an operator such that

$$
S f(s)=\sum_{k=1}^{\infty} a_{k}(s) f\left(\sigma_{k}(s)\right) \quad \text { and } \quad \alpha\|f\|_{1} \geq\|S f\|_{1} \geq\|f\|_{1}
$$

for every $f \in L^{1}$. Then there exists an operator $U: L^{1} \rightarrow L^{1}$, of the form

$$
U f(s)=c(s) f(\sigma(s)),
$$

such that

$$
\|S-U\| \leq 2\left(\alpha-\frac{1}{\alpha}\right)
$$

Proof. We consider, as before, $\Psi(f)=\left(\left|a_{n}(s)\right| f\left(\sigma_{n}(s)\right)\right)_{n \geq 1}$ as an operator into $L^{1}\left(c_{0}\right)$. Since by $(* *)$

$$
\alpha^{-1}\|\Psi(f)\|_{L^{1}\left(c_{0}\right)} \leq\|f\|_{L^{1}},
$$

there exists $\left(b_{k}\right)_{k}$ with $b_{k} \geq 0, \sum_{k} b_{k} \leq 1$, and

$$
\int_{0}^{1} \sum_{k=1}^{\infty}\left|a_{k}(s)\right| b_{k}(s) f\left(\sigma_{k}(s)\right) d s=\frac{1}{\alpha} \int_{0}^{1} f(s) d s .
$$

We may and do assume that $b_{1} \geq b_{2} \geq \cdots$. Therefore, for all $k \geq 2$,

$$
1-b_{k} \geq 1-b_{1} \geq b_{k}
$$


Since $\alpha\|f\|_{1} \geq\||S|(f)\|_{1}$, one has, for all $f \geq 0$,

$$
\begin{aligned}
\left(\alpha-\frac{1}{\alpha}\right)\|f\|_{1} & \geq \int_{0}^{1} \sum_{k=1}^{\infty}\left|a_{k}(s)\right|\left(1-b_{k}(s)\right) f\left(\sigma_{k}(s)\right) d s \\
& \geq \int_{0}^{1} \sum_{k=2}^{\infty}\left|a_{k}(s)\right| b_{k}(s) f\left(\sigma_{k}(s)\right) d s .
\end{aligned}
$$

Hence if we define

and

$$
V f(s)=\sum_{k=2}^{\infty} a_{k}(s) b_{k}(s) f\left(\sigma_{k}(s)\right)
$$

$$
\begin{aligned}
W f(s) & =\sum_{k=1}^{\infty} a_{k}(s) b_{k}(s) f\left(\sigma_{k}(s)\right) \\
& =a_{1}(s) b_{1}(s) f\left(\sigma_{1}(s)\right)+V f(s),
\end{aligned}
$$

one has, for all $f \geq 0$,

$$
\||S-W|(f)\|_{1}=\||S|(f)-|W|(f)\|_{1} \leq\left(\alpha-\frac{1}{\alpha}\right)\|f\|_{1},
$$

and also

$$
\||V|(f)\|_{1} \leq\left(\alpha-\frac{1}{\alpha}\right)\|f\|_{1},
$$

thus

$$
\|S-W\| \leq\left(\alpha-\frac{1}{\alpha}\right) \quad \text { and } \quad\|V\| \leq\left(\alpha-\frac{1}{\alpha}\right) .
$$

We let now $U=W-V$. We have:

$$
\|S-U\|=\|S-W+V\| \leq\|S-W\|+\|V\| \leq 2\left(\alpha-\frac{1}{\alpha}\right) .
$$

Proposition II.6. Let $U: L^{1} \rightarrow L^{1}$ be of the form

$$
U f(s)=c(s) f(\sigma(s)),
$$

and assume that for some $\alpha \geq 1$ we have, for all $f \in L^{1}$,

$$
\alpha\|f\|_{1} \geq\|U(f)\|_{1} \geq\|f\|_{1} .
$$


Then there exists an isometry $J$, from $L^{1}$ to $L^{1}$, such that $\|U-J\| \leq(\alpha-1)$.

Proof. By the Radon-Nikodym theorem, there exists $w$ such that, for all $f \in L^{1}$,

$$
\int_{0}^{1}|U|(f)(s) d s=\int_{0}^{1} w(s) f(s) d s .
$$

For all $f \geq 0$, one has

$$
\alpha \int_{0}^{1} f(s) d s \geq \int_{0}^{1} w(s) f(s) d s \geq \int_{0}^{1} f(s) d s .
$$

It follows that $1 \leq w(s) \leq \alpha$ for all $s$. We define

$$
J f(s)=\frac{c(s)}{w(\sigma(s))} f(\sigma(s))
$$

and compute

$$
\begin{aligned}
\|J f\|_{1} & =\int_{0}^{1}|J f(s)| d s=\int_{0}^{1} \frac{|c(s)|}{w(\sigma(s))}|f(\sigma(s))| d s \\
& =\int_{0}^{1}|c(s)|\left(\frac{|f|}{w}\right)(\sigma(s)) d s \\
& =\int_{0}^{1}|U|\left(\frac{|f|}{w}\right)(s) d s=\int_{0}^{1} w(s)\left(\frac{|f|}{w}\right)(s) d s=\|f\|_{1} .
\end{aligned}
$$

Hence $J$ is an isometry. Moreover, for any $s$, we have

$$
\mu_{s}^{|U-J|}=|c(s)|\left(1-\frac{1}{w(\sigma(s))}\right) \delta_{\sigma(s)},
$$

and since

$$
0 \leq 1-\frac{1}{w(s)} \leq 1-\frac{1}{\alpha}=\frac{\alpha-1}{\alpha}
$$

we have

$$
\|U-J\|=\||U-J|\| \leq \frac{\alpha-1}{\alpha}\|U\| \leq(\alpha-1) .
$$

From Theorem II.4, Proposition II.5, and Proposition II.6 we deduce the following quantitative improvement of Alspach's theorem ([1]). 
Theorem II.7. There is a function $\varphi(\alpha)$, with $\lim _{\alpha \rightarrow 1_{+}} \varphi(\alpha)=0$ and such that, if $T: L^{1} \rightarrow L^{1}$ satisfies for some $\alpha \geq 1$

$$
\alpha\|f\|_{1} \geq\|T f\|_{1} \geq\|f\|_{1} \quad \text { for all } f \in L^{1},
$$

then there is an isometry $J: L^{1} \rightarrow L^{1}$ such that, for all $f \in L^{1}$,

$$
\|T-J\| \leq \varphi(\alpha)
$$

Moreover, for $1 \leq \alpha \leq 1.08$ we can choose $\varphi(\alpha)=13(\alpha-1)$.

Proof. The first part clearly follows from Theorem II.4, Proposition II.5, and Proposition II.6. For the second part, apply Theorem II.4 to find an $S$ so that $\|T-S\| \leq \frac{17}{16}(\alpha-1)=\delta_{1}<1$. Then $S^{\prime}=S /\left(1-\delta_{1}\right)$ verifies $\|f\|_{1} \leq\left\|S^{\prime} f\right\|_{1} \leq \alpha_{1}\|f\|_{1}$, with

$$
\alpha_{1}=\frac{\delta_{1}+\alpha}{1-\delta_{1}} \leq 1.28 \quad \text { and } \quad\left\|T-S^{\prime}\right\| \leq \frac{\delta_{1}}{1-\delta_{1}}(1+\alpha) \leq 2.5(\alpha-1) .
$$

Now, Proposition II.5 gives an operator $U$ so that $\left\|S^{\prime}-U\right\| \leq 2\left(\alpha_{1}-\alpha_{1}^{-1}\right)=$ $\delta_{2}<1$; the operator $U^{\prime}=U /\left(1-\delta_{2}\right)$ verifies $\|f\|_{1} \leq\left\|U^{\prime} f\right\|_{1} \leq \alpha_{2}\|f\|_{1}$, with $\alpha_{2}=\left(\delta_{2}+\alpha_{1}\right) /\left(1-\delta_{2}\right)$, and we have

$$
\left\|S^{\prime}-U^{\prime}\right\| \leq \frac{\delta_{2}}{1-\delta_{2}}\left(1+\alpha_{1}\right) \leq 3.4(\alpha-1) .
$$

Finally, Proposition II.6 gives a $J$ so that $\left\|U^{\prime}-J\right\| \leq \alpha_{2}-1 \leq 6.8(\alpha-1)$.

Remark II.8. We give a proof of Theorem II.7 in the special case where $T=C_{\mu}$ is the convolution by a measure $\mu$ on $L^{1}(\mathbb{T})$. Suppose that $\|\mu\|=1$ and that $\|f * \mu\|_{1} \geq(1-\varepsilon)\|f\|_{1}$. Write the atomic part of $\mu$ as $\sum_{k=1}^{+\infty} a_{k} \delta_{x_{k}}$. We have

$$
\sum_{k=1}^{+\infty}\left|a_{k}\right| \leq\|\mu\|=1 .
$$

On the other hand, Wiener's theorem ([20], p. 42; [13], p. 415):

$$
\lim _{N \rightarrow+\infty} \frac{1}{2 N+1} \sum_{n=-N}^{N}|\hat{\mu}(n)|^{2}=\sum_{k=1}^{+\infty}\left|a_{k}\right|^{2}
$$


says that

$$
(1-\varepsilon) \leq \sum_{k=1}^{+\infty}\left|a_{k}\right|^{2},
$$

since

$$
|\widehat{\mu}(n)|=\left\|\mu * e_{n}\right\|_{1} \geq(1-\varepsilon),
$$

where $e_{n}(t)=e^{2 \pi i n t}$. It follows from (3) and (4) that there exists an index $K$ such that $\left|a_{K}\right| \geq(1-2 \varepsilon)$; thus the distance between $C_{\mu}$ and translation by $x_{K}$ is less than $4 \varepsilon$, and this concludes the proof.

There is an interesting link between Wiener's theorem and the equation (1) in the proof of Lemma II.1. Indeed, let $\mu$ be a measure on the Cantor group $G=\{-1,1\}^{\mathbb{N}}$, and let $\left(W_{k}\right)$ and $\left(D_{k}\right)$ be the Walsh functions and the elementary open sets with index $k \in\{-1,1\}^{[\mathrm{N}]}$. With this notation one has, by Parseval's formula,

$$
2^{-l} \sum_{k \in 2^{l}}\left(\int W_{k} d \mu\right)^{2}=\sum_{k \in 2^{l}} \mu\left(D_{k}\right)^{2}
$$

for any $l \geq 1$, and thus equation (1) appears, through the natural measure preserving isomorphism between $G$ and the unit interval, as the exact analogue for the Cantor group of Wiener's theorem. It would be nice to state a generalization of Wiener's theorem to arbitrary compact abelian groups.

III. Small subspaces of $L^{1}$. In this section, we introduce and study the notion of small subspace of $L^{1}$, which will be essential in the next section. In the sequel the notation $A \subseteq D$ will be used to say that there is a real number $k>0$ such that $k A \subseteq D$. If $X$ is a subspace of $L^{1}$, we denote by $C_{X}={\overline{B_{X}}}^{\tau_{m}}$ the closure of its unit ball for the topology $\tau_{m}$ of convergence in measure. Recall that Bukhvalov and Lozanovski ([4]) showed that $P\left(B_{X^{\perp \perp}}\right)=C_{X}$, where $P$ is the natural projection from $L^{1 * *}$ to $L^{1}$. We also denote, for $A \subseteq \Omega$,

$$
L^{1}(A)=\left\{f \in L^{1} \mid \mathbf{1}_{\Omega \backslash A} \cdot f=0 \text { a.e. }\right\} .
$$

Definition III.1. A subspace $X$ of $L^{1}(\Omega, m)$ is said to be small if there is no $A \subseteq \Omega$ of positive measure such that $B_{L^{1}(A)} \subseteq \mathbf{1}_{A} \cdot B_{X}$.

In other words, the projection $f \mapsto f \cdot \mathbf{1}_{A}$ never maps $X$ onto $L^{1}(A)$. It is easy to see (by lifting finite trees for instance) that reflexive subspaces of 
$L^{1}$ are small. However, smallness is not an intrinsic notion, but is related to the position of $X$ in $L^{1}$. Indeed, let $Q: \ell_{1} \rightarrow L^{1}$ be a quotient map, and $J: \ell_{1} \rightarrow L^{1}$ be an isometric embedding. Then the range of $(J, Q)$ : $\ell_{1} \rightarrow L^{1} \times L^{1}$ is isomorphic to $\ell_{1}$, but is not small. On the other hand, in $L^{1}([0,1] \times[0,1])$, the subspace of the functions which do not depend of the second coordinate is small, although it is isometric to $L^{1}$. We are now going to give some examples and properties of small subspaces.

It will be useful to know that small subspaces actually satisfy a formally stronger property.

Proposition III.2. If $X$ is a small subspace of $L^{1}$, there is no $E \subseteq \Omega$ of positive measure such that $B_{L^{1}(E)} \subseteq \mathbf{1}_{E} \cdot C_{X}$.

Proof. Assume that $B_{L^{1}(E)} \subseteq \mathbf{1}_{E} \cdot\left(k C_{X}\right)$, and let $\left\{x_{n}\right\}$ be dense in $B_{L^{1}(E)}$. There are $f_{n} \in k B_{X}$, and $E_{n} \subseteq E$, with $m\left(E_{n}\right) \leq 4^{-n} m(E)$, such that $\left|x_{n}-f_{n}\right| \leq 1 / n$ on $\left(E \backslash E_{n}\right)$. If now $A=E \backslash\left(\bigcup_{n \geq 1} E_{n}\right)$, we have $B_{L^{1}(A)} \subseteq$ $\mathbf{1}_{A} \cdot\left(k B_{X}\right)$, and so $X$ is not small.

The following notion is closely related to smallness. It should be however noted that, except in the proof of Proposition III.9, only the property stated in Proposition III.4 will actually be used.

Definition III.3. Let $T$ be an operator on $L^{1}$, written as

$$
T f(x)=\int_{0}^{1} f(s) d \mu_{x}(s) .
$$

$T$ will be called a strong Enflo operator if there exists a set of positive measure on which $\mu_{s}(\{s\}) \neq 0$.

In other words, $T$ has a "diagonal part". Such operators necessarily have an atomic part, and so are isomorphisms on some subspace $L^{1}(A)$ ([18], Theorem 5.5); this means that they are Enflo operators ([8], Theorem 4.1). Translations are Enflo operators but not strong. The proof of Theorem 5.4 in [18] essentially shows:

Proposition III.4. If $T$ is a strong Enflo operator, there exists a set A of positive measure such that

$$
B_{L^{1}(A)} \subseteq \mathbf{1}_{A} \cdot T\left(B_{L^{1}(A)}\right) .
$$


Indeed, if $T$ is a strong Enflo operator and $T^{a} f(s)=\sum_{n=1}^{+\infty} a_{n}(s) f\left(\left(\sigma_{n}(s)\right)\right.$ represents the atomic part of $T$, there exist an $n$ and a set $C$ of positive measure on which $a_{n}(s) \neq 0$ and $\sigma_{n}(s)=s$. It follows that, with the notation used in the proof of Theorem 5.4 of [18], $\sigma_{n}\left(B_{m, i} \cap B\right) \subseteq C_{m, i}$, and this set $A=B_{m, i} \cap B$ gives the conclusion.

Lemma III.5. Let $X$ be a subspace of $L^{1}(\Omega)$, and $T: L^{1}(A) \rightarrow L^{1}(\Omega)$ an operator, where $A \subseteq \Omega$. Then $T\left(B_{L^{1}(A)}\right) \subseteq \mathbf{1}_{A} \cdot C_{X}$ if and only if there exists an operator $\tilde{T}: L^{1}(A) \rightarrow X^{\perp \perp}$ such that $T=\mathbf{1}_{A} P \widetilde{T}$.

Proof. The sufficiency comes directly from Bukhvalov-Lozanovski's theorem $P\left(B_{X^{\perp \perp}}\right)=C_{X}$. To see the other direction, write $L^{1}(A)=\bigcup_{n \geq 1} L_{n}$ with $L_{n} \subseteq L_{n+1}$ and $L_{n}$ isometric to $\ell_{1}^{\operatorname{dim} L_{n}}$. Since $T\left(B_{L^{1}(A)}\right) \subseteq \mathbf{1}_{A} \cdot C_{X}$, there exist operators $T_{n}: L_{n} \rightarrow X$, such that $\left\|T_{n}\right\| \leq M$ and $d_{m}\left(\mathbf{1}_{A} T_{n} f, T f\right) \leq 2^{-n}$ for $f \in B_{L_{n}}$, where $d_{m}$ is a distance generating the topology $\tau_{m}$. Taking a $w^{*}$-limit of the $T_{n}$ 's along an ultrafilter, gives $\tilde{T}: L^{1}(A) \rightarrow X^{\perp \perp}$ such that $\mathbf{1}_{A} P \widetilde{T}=T$. Indeed, if $F \in L^{1 * *}$ is the $w^{*}$-limit of a bounded filter $\left(f_{\alpha}\right)_{\alpha}$ of functions in $L^{1}$, there exists a sequence $\left(c_{k}\right)_{k}$ of convex combinations of the $f_{\alpha}$ 's, such that $d_{m}\left(c_{k}, P(F)\right) \underset{k \rightarrow+\infty}{\longrightarrow} 0$ (see [16], Lemma IV.3.1).

We can now state the following characterization of small subspaces.

Proposition III.6. A subspace $X$ of $L^{1}$ is small if and only if no strong Enflo operator $T$ verifies $T\left(B_{L^{1}}\right) \subseteq C_{X}$.

Proof. Suppose that $T$ is a strong Enflo operator such that $T\left(B_{L^{1}}\right) \subseteq C_{X}$. Since, by Proposition III.4, we have $B_{L^{1}(A)} \subseteq \mathbf{1}_{A} \cdot T\left(B_{L^{1}(A)}\right)$ for some set $A$ of positive measure, we obtain $B_{L^{1}(A)} \subseteq \mathbf{1}_{A} \cdot C_{X}$, in contradiction with the smallness of $X$. Suppose now that $X$ is not small, and let $A$ be of positive measure and such that $B_{L^{1}(A)} \subseteq \mathbf{1}_{A} \cdot C_{X}$. By applying Lemma III.5 to the natural injection $j_{A}: L^{1}(A) \rightarrow L^{1}$, we obtain $\tilde{j}_{A}: L^{1}(A) \rightarrow X^{\perp \perp}$, such that $j_{A}=\mathbf{1}_{A} P \tilde{j}_{A}$. Defining $T f=P \tilde{j}_{A}\left(\mathbf{1}_{A} f\right)$ for $f \in L^{1}$, we get a strong Enflo operator $T: L^{1} \rightarrow L^{1}$, since, by uniqueness of the representation of $T$, we have $\mu_{s}^{T}=\delta_{s}$ for almost every $s \in A$, and we have also $T\left(B_{L^{1}(A)}\right) \subseteq C_{X}$.

Subspaces $X$ for which $C_{X}=B_{X}$, i.e., nicely placed subspaces (see [10], Definition 2), are of particular interest: if $X$ is nicely placed and no operator from $L^{1}$ to $X$ is strongly Enflo, then $X$ is small. Since, by Proposition III.4, 
the range of every strong Enflo operator contains an isomorphic copy of $L^{1}$, we get:

Proposition III.7. Every nicely placed subspace of $L^{1}$ which contains no subspace isomorphic to $L^{1}$ is small.

Note that the above condition is not necessary. For instance, the subspace of $L^{1}\left([0,1]^{2}\right)$ consisting of the functions which do not depend on the second variable is isometric to $L^{1}$ and nicely placed, but it is small. A special case of Proposition III.7 is:

Proposition III.8. For every nicely placed Riesz subset $\Lambda$ of $\mathbb{Z}, L_{\Lambda}^{1}(\mathbb{T})$ is small.

Recall that $\Lambda$ is a Riesz set if every measure whose Fourier transform is carried by $\Lambda$ is absolutely continuous, and it is nicely placed if $L_{\Lambda}^{1}$ is nicely placed in $L^{1}$ (see [11], Definition 1.4 or [16], Definition IV.4.2). Proposition III.8 follows from the fact that $\Lambda$ is a Riesz set if and only if $L_{\Lambda}^{1}(\mathbb{T})$ has the Radon-Nikodym Property ([27]). A stronger result will be shown below (Proposition III.10).

Proposition III.6 gives also a stability result.

Proposition III.9. Let $X$ and $Y$ be two small subspaces such that $X \cap Y=\{0\}$ and $X \oplus Y$ is closed. Then $X \oplus Y$ is small.

Proof. Let $T: L^{1} \rightarrow L^{1}$ be such that $T\left(B_{L^{1}}\right) \subseteq C_{X \oplus Y}$. Lemma III.5 with $A=\Omega$ gives $\tilde{T}: L^{1} \rightarrow(X \oplus Y)^{\perp \perp}=X^{\perp \perp} \oplus Y^{\perp \perp}$ such that $T=P \widetilde{T}$. Let $\pi: X^{\perp \perp} \oplus Y^{\perp \perp} \rightarrow X^{\perp \perp}$ be the projection. Writing $U=\pi \tilde{T}$ and $V=(1-\pi) \tilde{T}$, we have $T=P U+P V$, and $P U\left(B_{L^{1}}\right) \subseteq C_{X}$ and $P V\left(B_{L^{1}}\right) \subseteq C_{Y}$. By Proposition III.6, $P U$ and $P V$ cannot be strong Enflo operators, so $\mu_{s}^{P U}(\{s\})=$ $\mu_{s}^{P V}(\{s\})=0$ for almost all $s$. Hence $\mu_{s}^{T}(\{s\})=0$ for almost all $s$, and $T$ is not a strong Enflo operator. By Proposition III.6 again, we can conclude that $X \oplus Y$ is small.

We now examine the translation invariant case. A subset $\Lambda$ of $\mathbb{Z}$ is said to be semi-Riesz ([37]) if every measure $\sigma$ whose Fourier transform is carried by $\Lambda$ has no atomic part. Every Riesz set is semi-Riesz, and Wiener's theorem ([20], p. 42; [13], p. 415):

$$
\lim _{N \rightarrow+\infty} \frac{1}{2 N+1} \sum_{n=-N}^{N}|\hat{\sigma}(n)|^{2}=\sum_{t \in \mathbb{T}} \mid \sigma\left(\left.\{t\}\right|^{2}\right.
$$


shows that every set $\Lambda$ with density zero, that is

$$
d(\Lambda)=\lim _{N \rightarrow+\infty} \frac{\#(\Lambda \cap\{-N, \ldots, N\})}{2 N+1}=0,
$$

is semi-Riesz. The set $\Lambda=\left\{\sum_{k=1}^{n} \varepsilon_{k} 4^{k} \mid \varepsilon_{k}=-1,0,1, n \geq 1\right\}$ is therefore semi-Riesz and not Riesz ([37], p. 126). We have:

Proposition III.10. Let $\Lambda$ be a nicely placed subset of $\mathbb{Z}$. Then $L_{\Lambda}^{1}(\mathbb{T})$ is small if and only if $\Lambda$ is a semi-Riesz set.

Proof. Suppose that $L_{\Lambda}^{1}(\mathbb{T})$ is not small, and let $T: L^{1}(\mathbb{T}) \rightarrow L^{1}(\mathbb{T})$ be the operator constructed in the proof of Proposition III.6. We have readily

$$
\begin{cases}\mu_{s}^{T}(\{s\})=0 & \text { if } s \notin A, \\ \mu_{s}^{T}(\{s\})=1 & \text { if } s \in A .\end{cases}
$$

Let $\tau_{t}: L^{1}(\mathbb{T}) \rightarrow L^{1}(\mathbb{T})$ be the translation given by

$$
\tau_{t} f(u)=f(t+u)
$$

Since for every operator $U$ we have

$$
\mu_{s}^{\tau_{t}^{-1} U \tau_{t}}(\{s\})=\mu_{s-t}^{U}(\{s-t\})
$$

if we define $\tilde{T}: L^{1}(\mathbb{T}) \rightarrow L^{1}(\mathbb{T})$ by

$$
\tilde{T}=\int_{\mathbb{T}} \tau_{t}^{-1} T \tau_{t} d m(t)
$$

we have, for all $s$,

$$
\mu_{s}^{\widetilde{T}}(\{s\})=\int_{\mathbb{T}} \mu_{s-t}^{T}(\{s-t\}) d m(t),
$$

and so $\mu_{s}^{\widetilde{T}}(\{s\})=m(A)^{-1}$. Since $\tau_{t} \tilde{T}=\tilde{T} \tau_{t}$ for every $t \in \mathbb{T}$, there exists a measure $\sigma$ such that $\tilde{T}(f)=\sigma * f$. We have then $\sigma(\{0\})=m(A)^{-1}$, so the atomic part of $\sigma$ is non zero, although $\sigma \in \mathcal{M}_{\Lambda}$. Conversely, suppose that 
$L_{\Lambda}^{1}$ is small. Let $\sigma$ be a measure with spectrum in $\Lambda$, and with atomic part

$$
\sigma_{a}=\sum_{n=1}^{+\infty} a_{n} \delta_{x_{n}} .
$$

The convolution operator defined by $T f=\sigma * f$ maps $L^{1}(\mathbb{T})$ into $L_{\Lambda}^{1}$, so that $T\left(B_{L^{1}(\mathbb{T})}\right) \cong B_{L_{\Lambda}^{1}}$. On the other hand, its representing measure has the atomic part

$$
\mu_{s}^{T_{a}}=\sum_{n=1}^{+\infty} a_{n} \delta_{s-x_{n}} .
$$

Since $L_{\Lambda}^{1}$ is small and nicely placed, $T$ cannot be a strong Enflo operator, so $\mu_{s}^{T}(\{s\})=0$ almost everywhere. This is possible only if $x_{n} \neq 0$ or $a_{n}=0$. Considering now, for each $p \geq 1$, the translated measure $\sigma * \delta_{-x_{p}}$ (which still have its spectrum in $\Lambda$ ) instead of $\sigma$, gives that $a_{p}=0$, since its atomic part is $\sum_{n=1}^{+\infty} a_{n} \delta_{x_{n}-x_{p}}$. Hence $\sigma_{a}=0$, and we are done.

Example. Y. Meyer ([28], Theorem 6) showed that, if $\left(n_{k}\right)_{k \geq 1}$ grows fastly enough:

$$
n_{k+1}>6\left(n_{1}+\cdots+n_{k}\right) \text { and } \sum_{k=1}^{+\infty} \frac{n_{k}}{n_{k+1}}<+\infty,
$$

then the set

$$
\Lambda=\left\{\sum_{k=1}^{K} \varepsilon_{k} n_{k} \mid \varepsilon_{k}=-1,0,1, K \geq 1\right\}
$$

is closed for the Bohr topology; hence it is nicely placed. Since it has density zero, $L_{\Lambda}^{1}(\mathbb{T})$ is small, but $\Lambda$ is not a Riesz set, as the spectrum of a Riesz product. F. Parreau pointed out to us that the same conclusion holds for

$$
\Lambda=\left\{\sum_{k=1}^{K} \varepsilon_{k} 4^{k} \mid \varepsilon_{k}=-1,0,1, K \geq 1\right\} .
$$

We do not know whether $L_{\Lambda}^{1}$ contains a subspace isomorphic to $L^{1}$ whenever $\Lambda$ is the spectrum of a Riesz product.

For every subset $\Lambda$ of $\mathbb{Z}$, denote by $[\Lambda]$ the smallest nicely placed subset of $\mathbb{Z}$ containing $\Lambda$ (see [11], p. 306). [ $\Lambda$ ] is contained in the Bohr-closure of $\Lambda$ ([11], Corollary 2.6). 
Corollary III.11. If $L_{\Lambda}^{1}(\mathbb{T})$ is not small, $[\Lambda]$ contains a translate of the spectrum of a Riesz product.

Proof. If $L_{\Lambda}^{1}(\mathbb{T})$ is not small, $L_{[\Lambda]}^{1}(\mathbb{T})$ is a fortiori not small. Hence there is a measure $\sigma$ with spectrum in $[\Lambda]$ which has an atomic part, and Wiener's theorem shows that $\hat{\sigma}(n) \underset{|n| \rightarrow+\infty}{\longrightarrow} 0$. Host-Parreau's theorem $([17])$ ends the proof.

\section{Lifting of operators between quotients by small subspaces of} $L^{1}$. In this section, we show that operators between quotients of $L^{1}$ by small subspaces lift to operators on $L^{1}$. We begin with a special case, which is much easier than the general one. The following notion will be used:

Definition IV.1. The operator $T: L^{1} \rightarrow L^{1}$ is said to be a Daugavet operator if the Daugavet equation $\|I+\lambda T\|=1+\|T\|$ is fulfilled for every $\lambda$ with $|\lambda|=1$.

A. Plichko and M. Popov showed ([30], Section 9, Theorem 8) that every non-Enflo operator is Daugavet. In fact, more is true: $T$ is a Daugavet operator whenever it is not strongly Enflo. Indeed, denoting the representing measures of $T$ by $\mu_{s}, s \in[0,1]$, we have

$$
\|T\|=\sup _{E} \frac{1}{m(E)} \int_{0}^{1}\left|\mu_{s}\right|(E) d m(s),
$$

and

$$
\|I+\lambda T\|=\sup _{E} \frac{1}{m(E)} \int_{0}^{1}\left|\delta_{s}+\lambda \mu_{s}\right|(E) d m(s) .
$$

Since $T$ is not strongly Enflo, we have $\mu_{s}(\{s\})=0$ for almost all $s$, and the measures $\delta_{s}$ and $\mu_{s}$ are disjoint for these values. Hence, if for every $\varepsilon>0$ we choose a measurable set $E$ such that

$$
\|T\| \leq \frac{1}{m(E)} \int_{0}^{1}\left|\mu_{s}\right|(E) d m(s)+\varepsilon,
$$

we get, since we may assume that $s \in E$,

$$
1+\|T\| \leq \frac{1}{m(E)} \int_{0}^{1}\left|\delta_{s}+\lambda \mu_{s}\right|(E) d m(s)+\varepsilon \leq\|I+\lambda T\|+\varepsilon,
$$

which gives the result. D. Werner used a similar argument in ([36]). 
Proposition IV.2. Let $X$ and $Y$ be nicely placed subspaces of $L^{1}$. Assume that $X$ and $Y$ are small, and that

$$
\operatorname{dist}\left(L^{1} / X, L^{1} / Y\right)<2 .
$$

Then there exists an invertible operator $V: L^{1} \rightarrow L^{1}$ such that $V(X)=Y$. Moreover, for $0 \leq \alpha<1$, if

$$
\operatorname{dist}\left(L^{1} / X, L^{1} / Y\right)=1+\alpha,
$$

there is such a $V$ satisfying $\|V\| \cdot\left\|V^{-1}\right\| \leq(1+\alpha) /(1-\alpha)$.

Proof. Let $S$ be an isomorphism from $L^{1} / X$ onto $L^{1} / Y$ such that $\|S\|$. $\left\|S^{-1}\right\|<2$. By the lifting property of nicely placed subspaces (see [23], Theorem 1, [16], Proposition IV.2.12, or [19], Proposition 2.1), there exist $U$ and $V$ such that

$$
\left\{\begin{array}{l}
Q_{X} U=S^{-1} Q_{Y}, \\
Q_{Y} V=S Q_{X},
\end{array}\right.
$$

and such that $\|U\|=\left\|S^{-1}\right\|$ and $\|V\|=\|S\|$. Hence the following diagram is commutative:

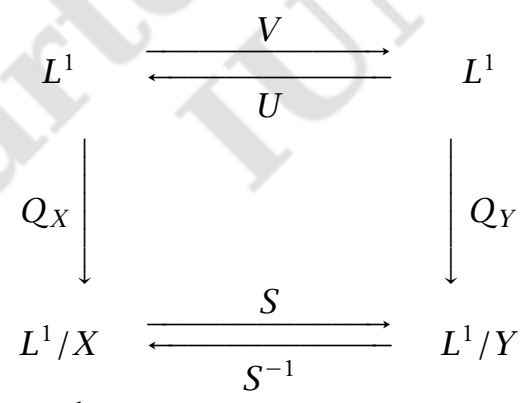

For every $f \in L^{1}$, we have

$$
Q_{Y} V U f=S Q_{X} U f=S S^{-1} Q_{Y} f=Q_{Y} f,
$$

hence

$$
(I-V U)\left(L^{1}\right) \subseteq Y,
$$

and similarly $(I-U V)\left(L^{1}\right) \subseteq X$. Since $X$ and $Y$ are nicely placed and small, the operators $I-U V$ and $I-V U$ are not strongly Enflo, and so are Daugavet operators. Therefore, we have

$$
\|I-U V\|=\|U V\|-1 \quad \text { and } \quad\|I-V U\|=\|V U\|-1 .
$$


Since $\|U V\| \leq\|U\| \cdot\|V\|=\|S\| \cdot\left\|S^{-1}\right\|<2$, it follows that $U$ and $V$ are invertible. Consequently $V(X)=Y$ since, for $f, g \in L^{1}$ such that $V(f)=g$, we have

$$
\begin{aligned}
g \in Y & \Leftrightarrow Q_{Y}(g)=0 \Longleftrightarrow Q_{Y} V(f)=0 \\
& \Leftrightarrow S Q_{X}(f)=0 \Longleftrightarrow Q_{X}(f)=0 \Longleftrightarrow f \in X .
\end{aligned}
$$

To conclude the proof, we observe that $\|V\|=\|S\|$ and

$$
V^{-1}=\left[\sum_{k=0}^{+\infty}(I-U V)^{k}\right] U
$$

Hence

$$
\left\|V^{-1}\right\| \leq \frac{\left\|S^{-1}\right\|}{2-\|S\| \cdot\left\|S^{-1}\right\|},
$$

since $\|U\|=\left\|S^{-1}\right\|$ and $\|I-U V\| \leq\|S\| \cdot\left\|S^{-1}\right\|$, and so $V$ works.

Remark. Note that Proposition IV.2 applies in particular to nicely placed subspaces which do not contain $L^{1}$ (by Proposition III.7). If $L^{1} / X$ and $L^{1} / Y$ are isometric, we have $\alpha=0$ and, by the above, $V$ is actually an invertible isometry of $L^{1}$.

We now give the general statement.

Theorem IV.3. Let $X$ and $Y$ be small subspaces of $L^{1}$, and suppose that there is an isomorphism $S: L^{1} / X \rightarrow L^{1} / Y$ such that $\|S\|$ and $\left\|S^{-1}\right\|$ are less than $1+\delta$, with $\delta<\frac{1}{25}$. Then, there exists an invertible operator $U: L^{1} \rightarrow L^{1}$ such that $\|U\| \cdot\left\|U^{-1}\right\| \leq(1+\delta) /(1-25 \delta)$, and $d_{\mathcal{H}}\left(U\left(B_{X}\right), B_{Y}\right) \leq 71 \delta /(1-25 \delta)$, where $d_{\mathcal{H}}$ denotes the Hausdorff distance.

Proof. Denote by $Q_{X}$ and $Q_{Y}$ the projections onto the respective quotient spaces. We first construct a bidual diagram

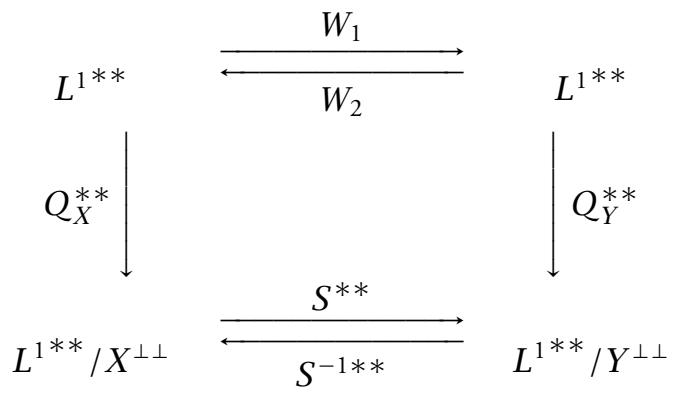


Write $L^{1}=\overline{\bigcup_{n \geq 1} L_{n}}$, where $\left(L_{n}\right)$ is an increasing sequence of finite-dimensional spaces isometric to $\ell_{1}^{\operatorname{dim}\left(L_{n}\right)}$. For all $n \geq 1$, there exists $T_{n}: L_{n} \rightarrow L^{1}$ such that

$$
Q_{Y} T_{n}=S Q_{X \mid L_{n}},
$$

and $\left\|T_{n}\right\| \leq(1+1 / n)\|S\|$.

Lemma IV.4. Let $\mathcal{U}$ be a free ultrafilter on the integers. There exists a $\left(w^{*}-w^{*}\right)$-continuous operator $W_{1}: L^{1 * *} \rightarrow L^{1 * *}$ such that

$$
\left\{\begin{array}{l}
\left\|W_{1}\right\| \leq\|S\|, \\
Q_{Y}^{* *} W_{1}=S^{* *} Q_{X}^{* *}, \\
W_{1}(f)=F,
\end{array}\right.
$$

for any $f \in \bigcup_{n \geq 1} L_{n}$ and $F=w^{*}-\lim _{u} T_{n}(f)$.

Proof. Define $\mathrm{w}_{1}: \bigcup_{n} L_{n} \rightarrow L^{1 * *}$ by

$$
\mathrm{w}_{1}(f)=w^{*}-\lim _{u} T_{n}(f)
$$

Clearly $\left\|\mathrm{w}_{1}\right\| \leq\|S\|$, and $\mathrm{w}_{1}$ can be extended to an operator $\overline{\mathrm{w}}_{1}: L^{1} \rightarrow$ $L^{1^{* *}}$. If now $\Pi:\left(L^{1}\right)^{(4)} \rightarrow L^{1^{* *}}$ denotes the canonical $\left(w^{*}-w^{*}\right)$-continuous

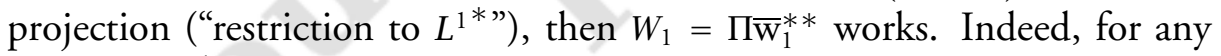
$g \in L^{1}$, one has

$$
Q_{Y}^{* *} \overline{\mathrm{w}}_{1}(g)=S Q_{X}(g),
$$

and the equation $Q_{Y}^{* *} W_{1}=S^{* *} Q_{X}^{* *}$ follows by $w^{*}$-continuity of all relevant operators.

We get of course, by the same token, an operator $W_{2}$ with $Q_{X}^{* *} W_{2}=$ $S^{-1 * *} Q_{Y}^{* *}$ and similar properties.

Lemma IV.5. Let $P: L^{1^{* *}} \rightarrow L^{1}$ be the natural projection. Then

$$
\left[P\left(I-W_{1} W_{2}\right)\right]\left(B_{L^{1 * *}}\right) \subseteq\left\|I-W_{1} W_{2}\right\|{\overline{B_{Y}}}^{\tau_{m}} .
$$

Proof. For any $h \in L^{1 * *}$, we have

$$
Q_{Y}^{* *} W_{1} W_{2} h=S^{* *} Q_{X}^{* *} W_{2} h=S^{* *} S^{-1 * *} Q_{Y}^{* *} h=Q_{Y}^{* *} h .
$$


Hence $Q_{Y}^{* *}\left(I-W_{1} W_{2}\right)=0$ and

$$
\left(I-W_{1} W_{2}\right)\left(B_{L^{1 * *}}\right) \subseteq\left\|I-W_{1} W_{2}\right\| B_{Y^{\perp \perp}}
$$

The result follows by Bukhvalov-Lozanovski’s theorem $P\left(B_{Y^{\perp \perp}}\right)={\overline{B_{Y}}}^{\tau_{m}}$.

Then, writing $T=P\left(I-W_{1} W_{2}\right)_{\mid L^{1}}$, it follows from Lemma IV.5, the smallness of $Y$, and Proposition III.6 that $T$ is not a strong Enflo operator; hence $-T$ satisfies the Daugavet equation, and we have

$$
\begin{aligned}
1+\left\|P\left(I-W_{1} W_{2}\right)_{\mid L^{1}}\right\| & =1+\|T\|=\|I-T\|=\left\|\left(P W_{1} W_{2}\right)_{\mid L^{1}}\right\| \\
& \leq\|S\| \cdot\left\|S^{-1}\right\| \leq 1+\delta .
\end{aligned}
$$

Therefore

$$
\left\|P\left(I-W_{1} W_{2}\right)_{\mid L^{1}}\right\| \leq \delta,
$$

and hence, for any $f \in L^{1}$, one has

$$
\left\|P\left(I-W_{1} W_{2}\right)(f)\right\|_{1} \geq(1-\delta)\|f\|_{1} .
$$

On the other hand, one also has

$$
\left\|W_{1} W_{2} f\right\|_{1} \leq\|S\| \cdot\left\|S^{-1}\right\|\|f\|_{1} \leq(1+\delta)\|f\|_{1} .
$$

Since $P$ is an L-projection, we have then

$$
\left\|W_{1} W_{2} f-P W_{1} W_{2} f\right\|_{1}=\left\|W_{1} W_{2} f\right\|_{1}-\left\|P W_{1} W_{2} f\right\|_{1} \leq 2 \delta\|f\|_{1} .
$$

It follows from this and (1) that

$$
\left\|\left(I-W_{1} W_{2}\right)_{\mid L^{1}}\right\| \leq 3 \delta .
$$

Since $W_{1}$ and $W_{2}$ are $w^{*}$-continuous, this gives

$$
\left\|I-W_{1} W_{2}\right\| \leq 3 \delta .
$$

An identical argument shows that

$$
\left\|I-W_{2} W_{1}\right\| \leq 3 \delta .
$$


Since $\delta<\frac{1}{3}$, (2) and (3) show that $W_{1} W_{2}$ and $W_{2} W_{1}$ are invertible. Hence $W_{1}$ and $W_{2}$ are themselves invertible.

Lemma IV.6. In the notation of Lemma IV.4, one has

$$
\|\|-\operatorname{diam}\left(L_{f}\right) \leq 6 \delta\left\|W_{2}^{-1}\right\|\|f\|_{1},
$$

where $L_{f}$ is the set of all $w^{*}$-cluster points of the $T_{n} f$ 's.

Proof. Since $\left\|f-W_{2} W_{1} f\right\|_{1} \leq 3 \delta\|f\|_{1}$ by (2), one has

$$
\left\|W_{2}^{-1} f-W_{1} f\right\|_{1} \leq 3 \delta\left\|W_{2}^{-1}\right\|\|f\|_{1} .
$$

By Lemma IV.4, any $F \in L_{f}$ can be written $F=W_{1}(f)$ for an appropriate $W_{1}$, and the conclusion follows.

Note that it follows, from (2) and (3), that

$$
\left\|W_{2}^{-1}\right\| \leq \psi(\delta)=\frac{1+\delta}{1-3 \delta} .
$$

Lemma IV.7. Let $Z$ be a Banach space such that $Z^{* *}=Z \oplus_{1} Z_{s}$. Let $\left(z_{n}\right)_{n}$ be a sequence in $Z$ and $L$ be the set of all its $w^{*}$-cluster points. If

$$
\|\|-\operatorname{diam}(L)=\varepsilon
$$

we have $\operatorname{dist}(l, Z) \leq \varepsilon$ for all $l \in L$.

Proof. Let $l \in L$. By translating if necessary, we may and do assume that $l \in Z_{s}$. Let $K=\left(B_{Z^{*}}, w^{*}\right)$. By ([6], Lemma III.2.4), we have

$$
\|l\|=\operatorname{dist}(l, Z)=\hat{l}=-l
$$

where $\hat{l}$ and $l$ are respectively the $u$.s.c. and the l.s.c. hulls of $l$. Thus

$$
w^{*}-\operatorname{Osc}\left(l_{\mid K}\right)(y)=2\|l\| .
$$

Pick $\alpha>0$. For any $n \geq 1$, let

$$
F_{n}=\left\{y \in K:\left|z_{k}(y)-z_{j}(y)\right| \leq \varepsilon+\alpha \text { for all } k, j \geq n\right\} .
$$


It follows from the assumption, that

$$
K=\bigcup_{n \geq 1} F_{n}
$$

Hence, by Baire's lemma, there is a non-void $w^{*}$-open set $U \subseteq K$ and an $N \geq 1$ such that $U \subseteq F_{N}$. It follows that, for all $y \in U$, we have

$$
\left|l(y)-z_{N}(y)\right| \leq \varepsilon+\alpha
$$

and since $z_{N}$ is $w^{*}$-continuous, this implies that for all $y \in U$ we have

$$
\operatorname{Osc}\left(l_{\mid K}\right)(y) \leq 2(\varepsilon+\alpha) \text {. }
$$

Hence $\operatorname{dist}(l, Z) \leq \varepsilon+\alpha$, which concludes the proof, since $\alpha>0$ was arbitrary. $p$

By Lemmas IV.6 and IV.7, we have

$$
\|P F-F\|=\operatorname{dist}\left(F, L^{1}\right) \leq 6 \delta \psi(\delta)\|f\|_{1}
$$

for every $F \in L_{f}$. Once we know this, it follows that one has

$$
\left\|\left(P W_{i}-W_{i}\right)_{\mid L^{1}}\right\| \leq 6 \delta \psi(\delta), \quad i=1,2 .
$$

We define now $U, V: L^{1} \rightarrow L^{1}$ by

$$
U=\left(P W_{1}\right)_{\mid L^{1}}, \quad V=\left(P W_{2}\right)_{\mid L^{1}}
$$

It follows from (1), (2), (3), and (4) that

$$
\|I-U V\| \leq \frac{22 \delta}{1-3 \delta} \quad \text { and } \quad\|I-V U\| \leq \frac{22 \delta}{1-3 \delta}
$$

so $U V$ and $V U$, and thus $U$ and $V$ as well, are invertible for $\delta<\frac{1}{25}$. Moreover, $\left\|U^{-1}\right\|$ and $\left\|V^{-1}\right\|$ are less than $1 /(1-25 \delta)$. We now observe that, since

$$
S Q_{X} f=Q_{Y}^{* *} W_{1} f \quad \text { for all } f \in L^{1},
$$

one has $W_{1}(X) \subseteq Y^{\perp \perp}$. It follows that $\operatorname{dist}\left(U f, Y^{\perp \perp}\right) \leq\left\|\left(P W_{1}-W_{1}\right)_{\mid L^{1}}\right\|$, so 
by $(4)$

$$
\operatorname{dist}(U f, Y) \leq 2 \operatorname{dist}\left(U f, Y^{\perp \perp}\right)<\frac{13 \delta}{1-3 \delta},
$$

for any $f \in B_{X}$. It follows that

$$
U\left(B_{X}\right) \subseteq B_{Y}+\frac{27 \delta}{1-3 \delta} B_{L^{1}}
$$

Since the same inclusion holds when we exchange $X$ and $Y$ and replace $U$ by $V$, we can find, for every $g \in B_{Y}$, an $f \in X$ such that $\|V g-f\|_{1} \leq$ $13 /(1-3 \delta)$. Letting $h=f /\|f\|_{1}$, we obtain

$$
\|g-U h\|_{1} \leq\left\|g-V^{-1} f\right\|_{1}+\left\|V^{-1}\right\|\|I-U V\|\|f\|_{1}+\|U\|\left(\|f\|_{1}-1\right),
$$

so

$$
B_{Y} \subseteq U\left(B_{X}\right)+\frac{71 \delta}{1-25 \delta} B_{L^{1}},
$$

and this finishes the proof of Theorem IV.3.

Let us say that $Y \subseteq L^{1}$ has the $\lambda$-lifting property if for any $T: L^{1} \rightarrow L^{1} / Y$, there exists $\widetilde{T}: L^{1} \rightarrow L^{1}$ such that $T=Q_{Y} \widetilde{T}$ and $\|\widetilde{T}\| \leq \lambda\|T\|$. If $Y$ is complemented in $Y^{* *}$, it has the lifting property (see [19], Lemma 2.1).

Corollary IV.8. Let $X$ and $Y$ be small subspaces of $L^{1}$ such that there is an isomorphism $S: L^{1} / X \rightarrow L^{1} / Y$, such that $\|S\| \cdot\left\|S^{-1}\right\| \leq 1+\delta$, and assume that $Y$ has the $\lambda$-lifting property. Then for $\delta \leq \delta(\lambda)$, there is $T: L^{1} \rightarrow L^{1}$, invertible, such that $T(X)=Y$, and

$$
\|T\| \cdot\left\|T^{-1}\right\| \leq 1+K(\lambda) \delta
$$

where $K(\lambda)$ only depends on $\lambda$.

Proof. Since $S Q_{X}=Q_{Y}^{* *} W_{1 \mid L^{1}}$, by (4) one has, for $\delta \leq \delta_{0}$,

$$
\left\|S Q_{X}-Q_{Y} U\right\|_{L^{1} \rightarrow L^{1} / Y} \leq C \delta .
$$

By assumption, there is $E: L^{1} \rightarrow L^{1}$ with

$$
\left\{\begin{array}{l}
\|E\| \leq C \lambda \delta, \\
Q_{Y} E=S Q_{X}-Q_{Y} U .
\end{array}\right.
$$


Now, if $T=U+E$, one has $S Q_{X}=Q_{Y} T$, and the conclusion follows, since this implies, when $T$ is invertible, that $T(X)=Y$.

Remark IV.9. There exist subspaces $X$ and $Y$ of infinite codimension in $c_{0}$ whose duals are isometric, but for which there is no invertible isometry of $\ell_{1}$ which maps $X^{\perp}$ onto $Y^{\perp}$. Indeed, every isometry from $\ell_{1}$ onto itself is $w^{*}$-continuous; hence there should exist an isometry of $c_{0}$ mapping $X$ onto $Y$. Therefore, it suffices to construct infinite codimensional isometric subspaces $X$ and $Y$ of $c_{0}$, in such a way that $X$ contains an element supported by a single integer, and that the elements of $Y$ are all supported by an even number of integers. Since invertible isometries of $c_{0}$ leave the size of the supports invariant, such $X$ and $Y$ cannot be mapped into each other by an isometry of all $c_{0}$. So Proposition IV.2 with $\ell_{1}$ instead of $L^{1}$ is false in full generality. That shows that it is essential that the measure space be purely non-atomic, at least for defining a proper notion of "small" subspace.

Remark IV.10. It is asked in [19] whether, for two infinite Sidon sets $S_{1}$ and $S_{2}$, the spaces $L_{\widetilde{S}_{1}}^{1}$ and $L_{\widetilde{S}_{2}}^{1}$ are isomorphic. It would be true if one could lift isomorphisms from $L^{1} / L_{\widetilde{S}_{1}}^{1}$ onto $L^{1} / L_{\widetilde{S}_{2}}^{1}$. But $L_{\widetilde{S}_{1}}^{1}$ and $L_{\widetilde{S}_{2}}^{1}$ are "large" subspaces of $L^{1}$, and the present techniques are apparently not applicable to this question.

V. Construction of a peculiar subspace of $L^{1}$. In Part II, we have seen a unified approach for Alspach's and Dor's theorems. In this section, we will see that the results of Part II have limitations. More precisely, we will construct a subspace $X$ of $L^{1}$ which will show that the nearness to isometries of the small into-isomorphisms from $L^{1}$ into $L^{1}$ (and of the small intoisomorphisms from finite dimensional subspaces of $L^{1}$ to $L^{1}$ ([21])) does not keep on to take place for general subspaces of $L^{1}$. Moreover, the same space will show that extension of isometries from a subspace of $L^{1}$ to the whole space $L^{1}$ (see [15], [26], [31]) is no longer true if we replace isometries by almost isometries. It shows also that Dor's Theorem B ([7]), which says that sequences of functions $f_{n}$ in $L^{1}$ which are equivalent to the canonical basis of $\ell_{1}$ are essentially supported by disjoint measurable sets, cannot be improved by replacing the $f_{n}$ 's by finite dimensional subspaces: our space $X$ is, for every $\varepsilon>0,(1+\varepsilon)$-isomorphic to a $\ell_{1}$-sum of finite dimensional spaces, but $\left\|\mathbb{E}^{S}-I d\right\|_{\mathcal{L}\left(X, L^{1}\right)} \geq 1$ for every $\sigma$-algebra $S$ generated by a measurable partition. Note also that in our terminology, the space $X$ we construct is small and nicely placed. We work in this section with real Banach spaces.

Theorem V.1. There exists a subspace $X$ of $L^{1}=L^{1}(\Omega, \Sigma, \mathbb{P})$ such that 
(a) For every isometry $T: X \rightarrow L^{1}$ there is a unique isometry $\tilde{T}: L^{1} \rightarrow L^{1}$ with $\tilde{T}_{\mid X}=T$;

(b) For every $\varepsilon>0$, there exists an isomorphism $T_{\varepsilon}: X \rightarrow L^{1}$, with $\left\|T_{\varepsilon}\right\|\left\|T_{\varepsilon}^{-1}\right\| \leq$ $1+\varepsilon$, but for which $\left\|T_{\varepsilon}-J\right\| \geq \frac{1}{2}$ for every isometry $J: X \rightarrow L^{1}$.

Before producing the proof, we note that (b) and Alspach's theorem (Theorem II.7) show that the operators $T_{\varepsilon}$ do not extend to $L^{1}$ with a proper control on the norm of the extension.

Proof. The subspace $X$ is constructed in a very similar way as the one given in [12], Example 4.1.1, but we work with the stable variables themselves instead of working with their absolute value.

The following lemma will be used.

Lemma V.2. Let $p \geq 1, \varepsilon>0$, and $k, l \geq 1$. There exists an $N \geq 1$ such that, for every subspace $F$ of $X=\ell_{p}^{N}$ with $\operatorname{dim} F \geq N-k$, there exist $l$ norm one vectors $y_{1}, \ldots, y_{l}$ of $\ell_{p}^{N}$, with disjoint support, such that $\operatorname{dist}\left(y_{j}, F\right) \leq \varepsilon$ $(1 \leq j \leq l)$.

Proof. Take $H$ bigger than $(1+2 / \varepsilon)^{k}$ and $N=H l$. Since $\operatorname{dim}(X / Z)=$ $k$, indices $i_{j}\left(H h<i_{2 h+1}<i_{2 h+2} \leq H(h+1), 0 \leq h \leq l\right)$ can be found such that dist $\left(e_{2 h+2}-e_{2 h+1}, F\right) \leq \varepsilon$, where $\left(e_{n}\right)_{1 \leq n \leq N}$ is the canonical basis of $\ell_{p}^{N}$.

Recall first some probabilistic facts (see [3], pp. 60-61). A random variable $Z$ is called $p$-stable if its characteristic function is $\hat{Z}(t) \stackrel{\text { def }}{=} \mathbb{E}\left(e^{i t Z}\right)=$ $e^{-c_{p}|t|^{p}}$, where $c_{p}$ is the normalization constant $\left(\|Z\|_{1}=1\right)$. Now, if $\left(Z_{n}\right)_{n \geq 1}$ is a sequence of independent $p$-stable variables, its linear span $X_{p}$ is isometric to $\ell_{p}$, and $Z_{1}, \ldots, Z_{n}, \ldots$ correspond to the usual vector basis of $\ell_{p}$. Since $c_{p} \underset{p \rightarrow 1}{\longrightarrow} 0$, for every $\varepsilon>0$, there is an $\alpha>0$ such that, if $1<p \leq \alpha$ :

$$
d_{\mathbb{P}}(f, 0) \leq \varepsilon \quad \text { for all } f \in B_{X_{p}},
$$

where $d_{\mathbb{P}}$ defines the convergence in probability. By the strong law of large numbers, we also have $\lim _{l \rightarrow+\infty}(1 / l) \sum_{j=1}^{l}\left|Z_{j}\right|=\mathbf{1}$ almost surely.

Now the space $X$ will be the closed linear span of the constant function 1 and a sequence of independent $p_{k}$-stable variables with $p_{k} \underset{k \rightarrow+\infty}{\rightarrow} 1$. More precisely, we choose inductively:

(1) a sequence $\left(p_{k}\right)_{k \geq 1}$ converging to 1 and a sequence $\left(\varepsilon_{k}\right)_{k \geq 1}$ converging to 0 such that

$$
d_{\mathbb{P}}(f, 0) \leq \varepsilon_{k} \quad \text { for all } f \in B_{X_{p_{k}}},
$$


where $X_{p_{k}}$ is the linear span of independent $p_{k}$-stable variables; these sequences are chosen so that the subspaces $X_{1}, X_{2}, \ldots$ constructed in the third step nearly create a $\ell_{1}$ sum. More explicitly, $\varepsilon_{k+1}$ is chosen so that the condition

$$
d_{\mathbb{P}}(f, 0) \leq \varepsilon_{k+1} \quad \text { for all } f \in B_{X_{p_{k+1}}}
$$

implies that, for $g \in X_{k+1}$ and $f \in X_{1}+\cdots+X_{k}$, we have

$$
\|f+g\|_{1} \geq\left(1-\frac{1}{2^{k+2}}\right)\left(\|f\|_{1}+\|g\|_{1}\right)
$$

(2) a sequence of integers $\left(l_{k}\right)_{k \geq 1}$ such that $\left|\left(1 / l_{k}\right) \sum_{j=1}^{l_{k}}\right| Y_{j}|-\mathbf{1}| \leq 1 / k$ almost surely for every independent $p_{k}$-stable variables $Y_{1}, \ldots, Y_{l_{k}}$;

(3) a sequence of integers $\left(N_{k}\right)_{k \geq 1}$ such that, if $X_{k}$ is the linear span of the independent $p_{k}$-stable variables $Z_{j}, j \in I_{k}\left(I_{k} \subseteq \mathbb{N},\left|I_{k}\right|=N_{k}\right)$, we can find, by Lemma V.2, for every finite dimensional subspace $F$ of $X_{k}, l_{k}$ independent $p_{k}$-stable variables $Y_{1}, \ldots, Y_{l_{k}}$ in $X_{k}$ such that \|\|$_{1}-\operatorname{dist}\left(Y_{j}, F\right) \leq 1 / 2^{k}$ for $1 \leq j \leq l_{k}$.

The sets $I_{1}, I_{2}, \ldots$ are chosen as successive intervals of $\mathbb{N}$, so we have $\mathbb{N}=$ $\bigcup_{k \geq 1} I_{k}$. All the $p_{k}$-stable variables for the different values of $k$ are independently chosen. Finally, we may and do suppose that the $\sigma$-algebra $\sigma(X)$ generated by $X$ is all $\Sigma$, since if not we replace $L^{1}(\Omega, \Sigma, \mathbb{P})$ by $L^{1}\left(\Omega, \sigma(X), \mathbb{P}_{\sigma(X)}\right)$. Since $1 \in X$, Hardin's theorem ([15], Corollary 4.3) now gives part (a) of the Theorem.

For the part $(b)$, we state two facts. The first one follows directly from the construction and the almost isometric embedding of finite dimensional subspaces of $L^{1}$ into $\ell_{1}$.

Fact 1. The Banach-Mazur distance from $X$ to the set of subspaces of $\ell_{1}$ is 1 .

It follows that for every $\varepsilon>0$, there exist an operator $T_{\varepsilon}: X \rightarrow L^{1}$ such that $\left\|T_{\varepsilon}\right\| \cdot\left\|T_{\varepsilon}^{-1}\right\| \leq 1+\varepsilon$ and a $\sigma$-algebra $S_{\varepsilon}$ generated by disjoint measurable parts of $\Omega$ such that $\mathbb{E}^{S_{\varepsilon}} T_{\varepsilon}=T_{\varepsilon}$.

Fact 2. For every sub- $\sigma$-algebra $S$ of $\Sigma$ generated by disjoint measurable sets of $\Omega$, one has

$$
\sup _{f \in B_{X}}\left\|\mathbb{E}^{S} f-f\right\|_{1} \geq 1
$$


Proof. Denote by $S_{1}, S_{2}, \ldots$ a measurable partition of $\Omega$ generating $S$, with $\mathbb{P}\left(S_{i}\right)>0$ for all $i$. For every $k \geq 1$, let

$$
F_{k}=\left\{f \in X_{k} \mid \int_{S_{i}} f d \mathbb{P}=0 \quad \text { for all } i \leq k\right\} .
$$

Let $\varepsilon>0$. By the third step of the construction, since $\operatorname{dim}\left(X_{k} / F_{k}\right) \leq k$, and since inf $f_{i \leq k} \mathbb{P}\left(S_{i}\right)>0$, there is an integer $k_{0}$ such that we can find, for every $k \geq k_{0}, l_{k}$ independent $p_{k}$-stable variables $Y_{1}, \ldots, Y_{l_{k}}$ in $X_{k}$ so that

$$
\left|\frac{1}{\mathbb{P}\left(S_{i}\right)} \int_{S_{i}} Y_{j} d \mathbb{P}\right| \leq \varepsilon \quad \text { for all } j \leq l_{k} \text {, all } i \leq k .
$$

Let $U_{k}=S_{1} \cup \cdots \cup S_{k}$. By $(*)$, one has

$$
\left\|\mathbb{E}^{S}\left(Y_{j}\right) \mathbf{1}_{U_{k}}\right\|_{1} \leq \varepsilon \quad \text { for all } j \leq l_{k} .
$$

But, by the step 2) of the construction, there is an integer $k_{1}$ such that

$$
\left\|\frac{1}{l_{k}} \sum_{j=1}^{l_{k}}\left|Y_{j}\right| \mathbf{1}_{U_{k}}\right\|_{1} \geq 1-\varepsilon
$$

for $k \geq k_{1}$, and then there is a $j_{k} \leq l_{k}$ such that

$$
\left\|\left|Y_{j_{k}}\right| \mathbf{1}_{U_{k}}\right\|_{1} \geq 1-\varepsilon .
$$

Hence

$$
\left\|Y_{j_{k}}-\mathbb{E}^{S} Y_{j_{k}}\right\|_{1} \geq\left\|Y_{j_{k}} \mathbf{1}_{U_{k}}-\left(\mathbb{E}^{S} Y_{j_{k}}\right) \mathbf{1}_{U_{k}}\right\|_{1} \geq 1-2 \varepsilon,
$$

and this proves the Fact 2 .

More generally, one has the following result:

Lemma V.3. For every isometry $J: X \rightarrow L^{1}$ and every $\sigma$-algebra generated by a measurable partition, one has

$$
\sup _{f \in B_{X}}\left\|\mathbb{E}^{S} J f-J f\right\|_{1} \geq 1 .
$$

Proof. From (a), one has an isometry $\widetilde{J}: L^{1} \rightarrow L^{1}$ which extends $J$. 
There are measurable functions $a, \sigma$ on $\Omega$ (see Section II) such that

$$
\widetilde{J} f(s)=a(s) f(\sigma(s)) .
$$

Since $\|a\|_{1}=\|\widetilde{J} \mathbf{1}\|_{1}=1$, the measure

$$
d Q=|a| d \mathbb{P}
$$

is a probability measure. Moreover,

$$
\int_{\Omega}|1+f(t)| d \mathbb{P}(t)=\int_{\Omega}|1+f(\sigma(s))| d Q(s) ;
$$

hence $f$ and $f(\sigma)$ have the same distribution ([15], Theorem 1.1); so $f(\sigma)$ is $p$-stable in $L^{1}(Q)$ whenever $f$ is $p$-stable in $L^{1}(\mathbb{P})$. Let $S$ be the $\sigma$-algebra generated by a measurable partition $\left(S_{i}\right)$ of $\Omega$, and let $S^{\prime}$ be a refinement of this partition, so that the sign of $a$ is constant on each $S_{i}^{\prime}(>0,<0$, or $=0)$. In particular, $\operatorname{supp}(a)=\bigcup\left\{S_{i}^{\prime} \mid a \neq 0\right.$ on $\left.S_{i}^{\prime}\right\}$.

Let $\varepsilon^{\prime}>0$. Reproducing the proof of the above Fact 2 in $L^{1}(Q)$, we obtain that, if $S_{i}^{\prime}(i \leq k)$ is disjoint from $\operatorname{supp}(a)$, there exist, for every $k \geq k_{0}, Y_{1}, \ldots, Y_{l_{k}} \in X_{k}$ such that

$$
\left|\frac{1}{Q\left(S_{i}^{\prime}\right)} \int_{S_{i}^{\prime}} Y_{j}(\sigma) d Q\right| \leq \varepsilon^{\prime} \quad \text { for all } i, \text { all } j \leq l_{k},
$$

that is

$$
\left|\frac{1}{Q\left(S_{i}^{\prime}\right)} \int_{S_{i}^{\prime}} Y_{j}(\sigma)\right| a|d \mathbb{P}| \leq \varepsilon^{\prime} \quad \text { for all } i, \text { all } j \leq l_{k} .
$$

But $a$ has a constant sign on $S_{i}^{\prime}$, so this writes

$$
\left|\frac{1}{Q\left(S_{i}^{\prime}\right)} \int_{S_{i}^{\prime}} Y_{j}(\sigma) a d \mathbb{P}\right| \leq \varepsilon^{\prime} \quad \text { for all } i, \text { all } j \leq l_{k} .
$$

Therefore

$$
\left|\frac{1}{\mathbb{P}\left(S_{i}^{\prime}\right)} \int_{S_{i}^{\prime}} Y_{j}(\sigma) a d \mathbb{P}\right| \leq \frac{Q\left(S_{i}^{\prime}\right)}{\mathbb{P}\left(S_{i}^{\prime}\right)} \varepsilon^{\prime} \quad \text { for all } i, \text { all } j \leq l_{k} .
$$

Consequently, for every $\varepsilon>0$, there is $k_{0}^{\prime}$ such that, for $k \geq k_{0}^{\prime}$, there exist 
$Y_{1}, \ldots, Y_{l_{k}} \in X_{k}$ such that

$$
\left|\frac{1}{\mathbb{P}\left(S_{i}^{\prime}\right)} \int_{S_{i}^{\prime}} Y_{j}(\sigma) a d \mathbb{P}\right| \leq \varepsilon \quad \text { for all } i, \text { all } j \leq l_{k}
$$

Now, since indices $i \leq k$ for which $S_{i}^{\prime}$ is disjoint from $\operatorname{supp}(a)$ do not matter, one has, as in the proof of Fact 2, for $k$ with $U_{k}=S_{1}^{\prime} \cup \cdots \cup S_{k}^{\prime} \in S$ :

$$
\left\|\mathbb{E}^{S} J\left(Y_{j}\right) \mathbf{1}_{U_{k}}\right\|_{1} \leq\left\|\mathbb{E}^{S^{\prime}} J\left(Y_{j}\right) \mathbf{1}_{U_{k}}\right\|_{1} \leq \varepsilon \quad \text { for all } j \leq l_{k} \text {. }
$$

On the other hand,

$$
\begin{array}{rl}
\frac{1}{l_{k}} \sum_{j=1}^{l_{k}}\left|Y_{j}(\sigma)\right| \underset{k \rightarrow+\infty}{\longrightarrow} 1 & \mathbb{P} \text {-a.s., } \\
& \text { hence } \frac{1}{l_{k}} \sum_{j=1}^{l_{k}}\left|J\left(Y_{j}\right)\right| \underset{k \rightarrow+\infty}{\rightarrow}|a| \quad Q-\text { a.s.. }
\end{array}
$$

Since $\|a\|_{L^{1}(\mathbb{P})}=1$, it follows that

$$
\varliminf_{k \rightarrow+\infty}\left\|\frac{1}{l_{k}} \sum_{j=1}^{l_{k}}\left|J\left(Y_{j}\right)\right| \mathbf{1}_{U_{k}}\right\|_{1} \geq 1 .
$$

There is therefore a $k_{1}$ such that, for every $k \geq k_{1}$, there is a $j_{k} \leq l_{k}$ such that

$$
\left\|J\left(Y_{j_{k}}\right) \mathbf{1}_{U_{k}}\right\|_{1} \geq 1-\varepsilon
$$

and so

$$
\left\|J\left(Y_{j_{k}}\right)-\mathbb{E}^{S} J\left(Y_{j_{k}}\right)\right\|_{1} \geq\left\|J\left(Y_{j_{k}}\right) \mathbf{1}_{U_{k}}-\mathbb{E}^{S} J\left(Y_{j_{k}}\right) \mathbf{1}_{U_{k}}\right\|_{1} \geq 1-2 \varepsilon,
$$

and this ends the proof of the lemma.

We can now finish the proof of Theorem V.1.

Let $\varepsilon>0, T_{\varepsilon}: X \rightarrow L^{1}$ with $\left\|T_{\varepsilon}\right\| \cdot\left\|T_{\varepsilon}^{-1}\right\| \leq 1+\varepsilon$, and $S_{\varepsilon}$ such that $\mathbb{E}^{S_{\varepsilon}} T_{\varepsilon}=T_{\varepsilon}$, as previously defined. For every isometry $J: X \rightarrow L^{1}$, we have

$$
\left\|\mathbb{E}^{S_{\varepsilon}} J-T_{\varepsilon}\right\|=\left\|\mathbb{E}^{S_{\varepsilon}} J-\mathbb{E}^{S_{\varepsilon}} T_{\varepsilon}\right\| \leq\left\|J-T_{\varepsilon}\right\|,
$$




$$
\left\|\mathbb{E}^{S_{\varepsilon}} J-J\right\| \leq\left\|\mathbb{E}^{S_{\varepsilon}} J-T_{\varepsilon}\right\|+\left\|T_{\varepsilon}-J\right\| \leq 2\left\|T_{\varepsilon}-J\right\| .
$$

But from Lemma V.3, $\left\|\mathbb{E}^{S_{\varepsilon}} J-J\right\| \geq 1$, and so $\left\|T_{\varepsilon}-J\right\| \geq 1 / 2$.

Remarks.

(1) There is a gap in the proof of the implication (iii) $\Rightarrow$ (iv) of Corollary 3.5 in [12], and we do not know whether one has (i) $\Rightarrow$ (iv) in that Corollary 3.5. Anyway, Theorem V.1 shows that one cannot replace the distance $d_{m}$ in [12], Corollary 3.5 (iv), by the \|\|$_{1}$ distance.

(2) We mention here that it follows from L. Schwartz's thesis ([34]; see [2], Theorem 4.2.5), that Müntz spaces $M_{1}\left(\left\{t^{n_{k}}\right\}\right)$ (with $\left.\sum n_{k}^{-1}<+\infty\right)$ are examples of subspaces of $L^{1}$ almost isometric to subspaces of $\ell_{1}$. The case $p>1$ has been noticed in [5] (see comments after Corollary 1.8).

The derivation of Lemma V.3 from Lemma V.2 can actually be put in a more general frame. This is the content of the next proposition.

Proposition V.4. Let $X_{1}$ and $X_{2}$ be isometric subspaces of $L^{1}\left(\Omega_{1}, \Sigma_{1}, \mu_{1}\right)$ and $L^{1}\left(\Omega_{2}, \Sigma_{2}, \mu_{2}\right)$ respectively. Suppose that $X_{1}$ contains the constant functions, and that, for some $\varepsilon>0$, there is a $\sigma$-algebra $\mathcal{A}$, generated by a measurable partition of $\Omega_{1}$, for which

$$
\left\|f-\mathbb{E}^{\mathcal{A}} f\right\|_{1} \leq \varepsilon\|f\|_{1} \quad \text { for all } f \in X_{1} .
$$

Then, for $\varepsilon^{\prime}>\varepsilon$, there is a $\left(\Sigma_{2} \otimes \mathcal{B}\right.$ or $)$-measurable partition of $\Omega_{2} \times[0,1]$, generating a $\sigma$-algebra $\mathcal{B}$, for which one has

$$
\left\|g-\mathbb{E}^{\mathcal{B}} \mathfrak{g}\right\|_{1} \leq \varepsilon^{\prime}\|g\|_{1} \quad \text { for all } g \in X_{2}
$$

In this statement, we identify $L^{1}\left(\Omega_{2}, \Sigma_{2}, \mu_{2}\right)$ to a subspace of

$$
L^{1}\left(\Omega_{2} \times[0,1], \Sigma_{2} \otimes \mathcal{B} \text { or }, \mu_{2} \otimes d t\right)=L^{1}\left(\tilde{\Omega}_{2}, \tilde{\Sigma}_{2}, \tilde{\mu}_{2}\right),
$$

by letting $\tilde{g}(\omega, t)=g(\omega)$ for $\omega \in \Omega_{2}, t \in[0,1]$, and $g \in L^{1}\left(\Omega_{2}, \Sigma_{2}, \mu_{2}\right)$.

Proof. Denote by $A_{1}, A_{2}, \ldots$ the partition generating $\mathcal{A}$, and by $\sigma_{1}$ and $\sigma_{2}$ the sub- $\sigma$-algebras of $\Sigma_{1}$ and $\Sigma_{2}$ generated by $X_{1}$ and $X_{2}$ respectively. Let $U$ be an isometry from $X_{1}$ onto $X_{2}$. Since $1 \in X_{1}$, Hardin's theorem ([15], Corollary 4.3) ensures the existence of an into-isometry

$$
T=\tilde{U}: L^{1}\left(\sigma_{1}\right) \rightarrow L^{1}\left(\Sigma_{2}\right)
$$


which extends $U$, and whose range is $L^{1}\left(\sigma_{2}\right)$. T can be written as $T f=a(f \circ$ $\tau)$, with $\tau$ an isomorphism between $\left(\Omega_{2}, \sigma_{2}\right)$ and $\left(\Omega_{1}, \sigma_{1}\right)$. Write $J f=f \circ \tau$. $J$ is positive, and so, since $\varphi_{n}=\mathbb{E}^{\sigma_{1}}\left(\mathbf{1}_{A_{n}}\right)$ verifies

$$
\varphi_{n} \geq 0 \text { and } \sum_{n \geq 1} \varphi_{n}=\mathbf{1}
$$

we have $\psi_{n}=J \varphi_{n} \geq 0$ and $\sum_{n \geq 1} \psi_{n}=\mathbf{1}$. Note that $v=|a| \mu_{2}$ is a probability measure and $J$ is an isometry from $L^{1}\left(\Omega_{1}, \sigma_{1}, \mu_{1}\right)$ to $L^{1}\left(\Omega_{2}, \sigma_{2}, v\right)$. We have:

Lemma V.5. If $\psi_{n}$ are positive $\sigma_{2}$-measurable functions with $\sum_{n \geq 1} \psi_{n}=\mathbf{1}$, there is a measurable partition in sets $B_{1}, B_{2}, \ldots \in \sigma_{2} \otimes \mathcal{B}$ or, such that $\widetilde{\psi}_{n}=$ $\mathbb{E}^{\sigma_{2}}\left(\mathbf{1}_{B_{n}}\right)$, for $n \geq 1$.

Proof. Set

$$
B_{n}=\left\{(\omega, t) \mid \sum_{k=1}^{n-1} \psi_{k}(\omega) \leq t<\sum_{k=1}^{n} \psi_{k}(\omega)\right\} .
$$

We have for every $B \in \sigma_{2}$ :

$$
\begin{aligned}
\int_{\widetilde{B}} \mathbb{E}^{\sigma_{2}} \mathbf{1}_{B_{n}} & =\int_{\widetilde{B}} \mathbf{1}_{B_{n}}=(\nu \otimes d t)\left(B_{n} \cap \widetilde{B}\right) \\
& =\int_{B} m\left[B_{n}(\omega)\right] d v(\omega)=\int_{B} \psi_{n}(\omega) d v(\omega),
\end{aligned}
$$

so $\widetilde{\psi}_{n}=\mathbb{E}^{\sigma_{2}} \mathbf{1}_{B_{n}}$.

We may suppose now that $\tilde{a}$ has a constant sign on each $B_{n}$. Indeed, since $\tilde{a}$ is $\sigma_{2}$-measurable and $\tau$ is an isomorphism from $\left(\Omega_{2}, \sigma_{2}\right)$ onto $\left(\Omega_{1}, \sigma_{1}\right)$, we have, if $B_{n}^{+}=B_{n} \cap\{\tilde{a} \geq 0\}$ and $B_{n}^{-}=B_{n} \cap\{\tilde{a}<0\}$,

$$
\begin{aligned}
\mathbb{E}^{\sigma_{2}}\left(\mathbf{1}_{B_{n}^{+}}\right) & =\mathbf{1}_{\{\tilde{a} \geq 0\}} \mathbb{E}^{\sigma_{2}}\left(\mathbf{1}_{B_{n}}\right)=\mathbf{1}_{\{\tilde{a} \geq 0\}} \tilde{\psi}_{n} \\
& =\mathbf{1}_{\{\tilde{a} \geq 0\}}\left(\tilde{\varphi}_{n} \circ \boldsymbol{T}\right)=\widetilde{\mathbb{E}}^{\sigma_{1}}\left(\mathbf{1}_{\left\{a \circ \tau^{-1} \geq 0\right\}} \mathbf{1}_{A_{n}}\right) \circ \boldsymbol{\tau},
\end{aligned}
$$

so we may cut each $A_{n}$ with the sets $\left\{a \circ \tau^{-1} \geq 0\right\}$ and $\left\{a \circ \tau^{-1}<0\right\}$, and the $B_{n}^{+}$'s and the $B_{n}^{-}$'s will correspond to the $A_{n}^{+}$'s and the $A_{n}^{-}$'s. Now, letting

$$
a_{n}(f)=\frac{1}{\mu_{1}\left(A_{n}\right)} \int_{A_{n}} f d \mu_{1}
$$


one has

$$
\begin{aligned}
\mu_{1}\left(A_{n}\right) & =\int_{\Omega_{1}} \mathbf{1}_{A_{n}} d \mu_{1}=\int_{\Omega_{1}} \varphi_{n} d \mu_{1}=\left\|\varphi_{n}\right\|_{1}=\left\|\psi_{n}\right\|_{L^{1}(v)} \\
& =\int_{\Omega_{2}} \psi_{n} d v=\int_{\widetilde{\Omega}_{2}} \mathbf{1}_{B_{n}} d \tilde{v}=\tilde{v}\left(B_{n}\right)
\end{aligned}
$$

and

$$
\begin{aligned}
& \int_{A_{n}} f d \mu_{1}=\int_{\Omega_{1}} f \mathbf{1}_{A_{n}} d \mu_{1}=\int_{\Omega_{1}} f \varphi_{n} d \mu_{1}=\int_{\Omega_{2}} J\left(f \varphi_{n}\right) d v \\
& \text { since } J f=f \circ \tau, \text { and } \mu_{1}=\tau^{*}(v), \\
&=\int_{\Omega_{2}}(J f) \psi_{n} d v=\int_{B_{n}} \tilde{J} f d \tilde{v} .
\end{aligned}
$$

Hence

$$
a_{n}(f)=\frac{1}{\tilde{v}\left(B_{n}\right)} \int_{B_{n}} \tilde{J} f d \tilde{v},
$$

and then, denoting by $\mathcal{B}$ the $\sigma$-algebra generated by $B_{1}, B_{2}, \ldots$,

$$
\begin{aligned}
\left\|\tilde{J} f-\mathbb{E}^{\mathcal{B}}(\tilde{J} f)\right\|_{L^{1}(\tilde{v})} & \left.=\sum_{n \geq 1} \int_{B_{n}}\left|\tilde{J} f-a_{n}(f) \mathbf{1}\right| d \tilde{v}=\sum_{n \geq 1} \int_{B_{n}} \mid \tilde{f} \circ \tau-a_{n}(f) \mathbf{1}\right) \mid d \tilde{v} \\
& =\sum_{n \geq 1} \int_{\Omega_{2}}\left|f \circ \boldsymbol{\tau}-a_{n}(f) \mathbf{1}\right| \psi_{n} d v \\
& =\sum_{n \geq 1} \int_{\Omega_{2}}\left|f \circ \boldsymbol{\tau}-a_{n}(f) \mathbf{1}\right|\left(\varphi_{n} \circ \tau\right) d v \\
& =\sum_{n \geq 1} \int_{\Omega_{1}}\left|f-a_{n}(f) \mathbf{1}\right| \varphi_{n} d \mu_{1}=\sum_{n \geq 1} \int_{A_{n}}\left|f-a_{n}(f) \mathbf{1}\right| d \mu_{1} \\
& =\left\|f-\mathbb{E}^{\mathcal{A}} f\right\|_{1} \leq \varepsilon\|f\|_{1} .
\end{aligned}
$$

Now, going back to $T$, we refine $\mathcal{B}$ in such a way that $\left\|\tilde{a}-\mathbb{E}^{\mathcal{B}} \tilde{a}\right\|_{L^{1}\left(\tilde{\mu}_{2}\right)} \leq$ $\alpha=\varepsilon^{\prime}-\varepsilon$. We keep the inequality $\left\|\tilde{J} f-\mathbb{E}^{\mathcal{B}}(\tilde{J} f)\right\|_{L^{1}(\tilde{v})} \leq \varepsilon\|f\|_{1}$. Moreover, this refinement can be made by taking the intersection with $\sigma_{2}$-measurable sets, so, as above, the new $B_{n}$ 's will correspond to an appropriate refinement of the $A_{n}$ 's. Set $\varepsilon_{n}=1$ if $a \geq 0$ on $B_{n}$, and $\varepsilon_{n}=-1$ if $a<0$ on $B_{n}$. One has

$$
\int_{B_{n}} \tilde{a} d \tilde{\mu}_{2}=\varepsilon_{n} \int_{\Omega_{2}}|a| \psi_{n} d \mu_{2}=\varepsilon_{n} \int|a|\left(\varphi_{n} \circ \tau\right) d \mu_{2}=\varepsilon_{n} \int_{\Omega_{2}}\left|T \varphi_{n}\right| d \mu_{2}
$$




\section{Operators Between Subspaces and Quotients of $L^{1}$$$
=\varepsilon_{n}\left\|T \varphi_{n}\right\|_{L^{1}\left(\mu_{2}\right)}=\varepsilon_{n}\left\|\varphi_{n}\right\|_{L^{1}\left(\mu_{1}\right)}=\varepsilon_{n} \mu_{1}\left(A_{n}\right),
$$

and so

$$
\mathbb{E}^{\mathcal{B}} \tilde{a}=\sum_{n \geq 1} \varepsilon_{n} \frac{\mu_{1}\left(A_{n}\right)}{\widetilde{\mu}_{2}\left(B_{n}\right)} \mathbf{1}_{B_{n}}
$$

Now

$$
\begin{aligned}
& \left\|\tilde{T} f-\mathbb{E}^{\mathcal{B}} \tilde{T} f\right\|_{L^{1}\left(\tilde{\mu}_{2}\right)} \\
& =\int_{\tilde{\Omega}_{2}}\left|\tilde{a}(\tilde{f} \circ \tau)-\sum_{n \geq 1}\left(\frac{1}{\tilde{\mu}_{2}\left(B_{n}\right)} \int_{B_{n}} \tilde{a}(\tilde{f} \circ \tau)\right) \mathbf{1}_{B_{n}}\right| d \tilde{\mu}_{2} \\
& \leq \int_{\widetilde{\Omega}_{2}}\left|\tilde{a}(\tilde{f} \circ \tau)-\sum_{n \geq 1}\left(\frac{\tilde{a}}{\mu_{1}\left(A_{n}\right)} \int_{B_{n}}|\tilde{a}|(\tilde{f} \circ \tau) d \tilde{\mu}_{2}\right) \mathbf{1}_{B_{n}}\right| d \tilde{\mu}_{2} \\
& +\int_{\tilde{\Omega}_{2}} \mid \sum_{n \geq 1}\left(\frac{\tilde{a}}{\mu_{1}\left(A_{n}\right)} \int_{B_{n}}|\tilde{a}|(\tilde{f} \circ \tau) d \tilde{\mu}_{2}\right) \mathbf{1}_{B_{n}} \\
& -\sum_{n \geq 1}\left(\frac{1}{\tilde{\mu}_{2}\left(B_{n}\right)} \int_{B_{n}} \tilde{a}(\tilde{f} \circ \tau) d \tilde{\mu}_{2}\right) \mathbf{1}_{B_{n}} \mid d \tilde{\mu}_{2} \\
& \leq \int_{\widetilde{\Omega}_{2}}\left|\tilde{f} \circ \tau-\sum_{n \geq 1}\left(\frac{1}{\tilde{v}\left(B_{n}\right)} \int_{B_{n}}(\tilde{f} \circ \tau) d \tilde{v}\right) \mathbf{1}_{B_{n}}\right| d \tilde{v} \\
& +\int_{\widetilde{\Omega}_{2}} \sum_{n \geq 1}\left(\frac{1}{\mu_{1}\left(A_{n}\right)} \int_{B_{n}}|\tilde{a}|(\tilde{f} \circ \tau) d \tilde{\mu}_{2}\right)\left(\tilde{a}-\varepsilon_{n} \frac{\mu_{1}\left(A_{n}\right)}{\tilde{\mu}_{2}\left(B_{n}\right)}\right) \mathbf{1}_{B_{n}} d \tilde{\mu}_{2} \\
& \leq\left\|\tilde{J} f-\mathbb{E}^{\mathcal{B}} \tilde{J} f\right\|_{L^{1}(\tilde{v})}+\int_{\widetilde{\Omega}_{2}} \sum_{n \geq 1}\|f\|_{1}\left|\tilde{a}-\varepsilon_{n} \frac{\mu_{1}\left(A_{n}\right)}{\widetilde{\mu}_{2}\left(B_{n}\right)}\right| \mathbf{1}_{B_{n}} d \tilde{\mu}_{2} \\
& \leq \varepsilon\|f\|_{1}+\|f\|_{1} \sum_{n \geq 1} \int_{B_{n}}\left|\tilde{a}-\varepsilon_{n} \frac{\mu_{1}\left(A_{n}\right)}{\widetilde{\mu}_{2}\left(B_{n}\right)}\right| d \tilde{\mu}_{2} \\
& \leq \varepsilon\|f\|_{1}+\|f\|_{1}\left\|\tilde{a}-\mathbb{E}^{\mathcal{B}} \tilde{a}\right\|_{L^{1}\left(\tilde{\mu}_{2}\right)} \leq(\varepsilon+\alpha)\|f\|_{1}=\varepsilon^{\prime}\|f\|_{1} .
\end{aligned}
$$

\section{Remarks.}

(1) A slight variation in Proposition V.4 is: set $C_{n}=\tau^{-1}\left(A_{n}\right)$. The $C_{n}$ 's are in general not in $\Sigma_{2}$, but if $C$ is the $\sigma$-algebra generated by them, the map $\tau: \Omega_{2} \rightarrow \Omega_{1}$ is $\left(\sigma_{2} \vee C-\sigma_{1} \vee \mathcal{A}\right)$-bi-measurable (see in [14] a description of these $\sigma$-algebras), and $\tilde{\mu}_{2}(B)=\mu_{1}[\tau(B)]$ defines a measure on $\left(\Omega_{2}, \sigma_{2} \vee C\right)$, whose restriction to $\sigma_{2}$ is equal to the restriction of $\mu_{2}$ to $\sigma_{2}$. One has then an isometry $\tilde{J}: L^{1}\left(\Omega_{1}, \sigma_{1} \vee \mathcal{A}, \mu_{1}\right) \rightarrow$ $L^{1}\left(\Omega_{2}, \sigma_{2} \vee C, \tilde{\mu}_{2}\right)$, defined by $\widetilde{J}(f)=f \circ \tau$, such that $\tilde{J} X_{1}=J X_{1}$. It follows that $\left\|J f-\mathbb{E}^{C} J f\right\|_{1} \leq \varepsilon\|f\|_{1}$. It would be interesting to find an 
intrinsic characterization of these spaces. This problem may be connected to the fact that, though there are sufficient conditions to have uniform convergence of martingales (see [29]), it seems there is at the present time no available necessary condition.

(2) Since the space $X$ and $T_{\varepsilon}(X)$ are small nicely placed subspaces (by Proposition III.7), it follows from Theorem V.1 and Proposition IV.2 that there is $\alpha>0$ such that the quotient spaces $L^{1} / X$ and $L^{1} / T_{\varepsilon}(X)$ have Banach-Mazur distance greater than $(1+\alpha)$ for all $\varepsilon>0$. This implies of course that the operators $T_{\varepsilon}$ do not extend to isomorphisms $U$ of $L^{1}$ such that the norms of $U$ and its inverse are small.

\section{REFERENCES}

[1] D. E. AlsPaCh, Small into isomorphisms on Lp spaces, Illinois J. Math 27 (1983), 300-314.

[2] P. Borwein \& T. ERdelyi, Polynomials and Polynomial Inequalities, Graduate Texts in Mathematics, Vol. 161, Springer-Verlag, New York.

[3] J. Bourgain \& H. P. Rosenthal, Martingales valued in certain subspaces of $L^{1}$, Israel J. Math. 37 (1980), 54-75.

[4] A. V. Bukhvalov \& G. LozanovskiI, On sets closed in measure, Trans. Moscow Math. Soc. 2 (1978), 127-148.

[5] F. Delbaen, H. Jarchow \& A. Pelczyński, Subspaces of $L_{p}$ isometric to subspaces of $\ell_{p}$, Positivity 2 (1998), 339-367.

[6] R. Deville, G. Godefroy \& V. Zizler, Smoothness and Renormings in Banach Spaces, Pitman Monographs in Pure and Applied Mathematics, Vol. 64, Pitman, 1993.

[7] L. E. Dor, On projections in $L^{1}$, Annals of Math. 102 (1975), 463-474.

[8] P. ENFlo \& T. W. STARbird, Subspaces of $L^{1}$ containing $L^{1}$, Studia Math. 65 (1979), 203-225.

[9] H. FAKHOURY, Représentations d'opérateurs à valeurs dans $L^{1}(X, \Sigma, \mu)$, Math. Ann. 240 (1979), 203-212.

[10] G. Godefroy, Sous-espaces bien disposés de L $L^{1}$-Applications, Trans. Amer. Math. Soc. 286 (1984), 227-249.

[11] - On Riesz subsets of abelian discrete groups, Israël J. Math. 61 (1988), 301-331.

[12] G. Godefroy, N. J. Kalton \& D. LI, On subspaces of $L^{1}$ which embed into $\ell_{1}$, Journal für die reine und angew. Math. 471 (1996), 43-75.

[13] C. C. Graham \& O. C. MCGehee, Essays in Commutative Harmonic Analysis, SpringerVerlag, 1979.

[14] A. Hanen \& J. Neveu, Atomes conditionnels d’un espace de probabilité, Acta Math. Acad. Sci. Hung. 17 (1966), 443-449.

[15] C D. Hardin JR., Isometries on subspaces of $L^{p}$, Indiana Univ. Math. J. 30 (1981), $449-465$.

[16] P. Harmand, D. Werner \& W. Werner, $M$-ideals in Banach spaces and Banach algebras, Lecture Notes in Math., vol. 1547, Springer-Verlag, 1993.

[17] B. Host \& F. PARREAU, Sur les mesures dont la transformée de Fourier ne tend pas vers zéro à l'infini, Colloq. Math. 41 (1979), 285-289. 
[18] N. J. Kalton, The Endomorphisms of $L_{p}(0 \leq p \leq 1)$, Indiana Univ. Math. J. 27 (1978), 353-381.

[19] N. J. KALtON \&A. PELCZYŃSKI, Kernels of surjections from $L_{1}$-spaces with an application to Sidon sets, Math. Ann. 309 (1997), 135-158.

[20] Y. Katznelson, An introduction to Harmonic Analysis, Wiley, 1976.

[21] A. L. Koldobskil, Convolution metric in the space of measures and $\varepsilon$-isometries in $L_{p}$, J. Soviet Math. 44 (1989), 852-855.

[22] E. LACEY, The Isometric Theory of Classical Banach Spaces, Springer-Verlag, 1974.

[23] D. LI, Lifting properties for some quotients of $L^{1}$-spaces and other spaces $L$-summand in their bidual, Math. Z. 199 (1988), 321-329.

[24] J. Lindenstrauss \& H. P. Rosenthal, Automorphisms in $c_{0}, \ell_{1}$ and $m$, Israel J. Math. 7 (1969), 227-239.

[25] J. Lindenstrauss \& L. Tzafriri, Classical Banach Spaces I and II, Classics in Math., Springer, 1996.

[26] W. LUSKY, Some consequences of Rudin's paper " $L_{p}$-Isometries and equimeasurability", Indiana Univ. Math. J. 27 (1978), 859-866.

[27] F. Lust-Piquard, Ensembles de Rosenthal et ensembles de Riesz, Comptes Rendus Acad. Sciences (Paris) Série A 282 (1976), 833-835.

[28] Y. Meyer, Spectres des mesures et mesures absolument continues, Studia Math. 30 (1968), 87-99.

[29] J. Neveu, Note on the tightness of the metric on the set of complete sub $\sigma$-algebras on a probability space, Ann. Math. Statistics 43 (1972), 1369-1371.

[30] A. M. Plichко \& M. M. POPOV, Symmetric function spaces on atomless probability spaces, Dissertationes Math. 306 (1990).

[31] A. I. Plotkin, Continuation of $L^{p}$ isometries, J. Soviet Math. 2 (1974), 143-165.

[32] H. P. Rosenthal, Embeddings of $L^{1}$ in $L^{1}$, Contemp. Math. 26 (1984), 335-349.

[33] G. Schechtman, Almost isometric $L_{p}$ subspaces of $L_{p}(0,1)$, Journal London Math. Soc. 20 (1979), 516-528.

[34] L. SCHWARTZ, Étude des sommes d'exponentielles, Hermann, 1943.

[35] M. Talagrand, The three-space problem for $L^{1}$, J. Amer. Math. Soc. 3 (1990), 9-29.

[36] D. Werner, An elementary approach to the Daugavet equation, Proc. Conf. Columbia 1994, Lecture Notes in Pure and Applied Mathematics 175 (1996), 449-454, Interaction Between Functional Analysis, Harmonic Analysis, and Probability (N. J. Kalton, E. Saab, \& S. Montgomery-Smith, eds.), M. Dekker.

[37] D. Werner, The Daugavet equation for operators on function spaces, J. Funct. Anal. 143 (1997), 117-128.

G. Godefroy

Université Pierre et Marie Curie

Equipe d'Analyse, Tour 46-0, Case 186, 4 place Jussieu

F-75252 Paris Cedex 05, FRANCE

EMAIL: gig@ccr.jussieu.fr 
N. J. KaLTON

Department of Mathematics

University of Missouri-Columbia

Columbia, MO 65211 U. S. A.

EMAIL: nigel@math.missouri.edu

D. LI

Faculté des Sciences Jean Perrin

Université d'Artois, Pôle de Lens

Rue Jean Souvraz, SP 18

F-62307 Lens Cedex, FRANCE

EMAIL: daniel.li@euler.univ-artois.fr

Subject Classification: Primary: 46A22, 46B20, 46B25, secondary: 43A15, 43A46, 60E07. KEYWORDS: Integral representation of operators on $L^{1}$, small into-isomorphisms of $L^{1}$, complemented subspaces of $L^{1}$, isometries of $L^{1}$, small subspaces of $L^{1}$, nicely placed subspaces of $L^{1}$, semi-Riesz sets, strong Enflo operators, Daugavet operators, lifting of operators, $p$-stable random variables.

Submitted: February 3rd, 1999, revised: November 22nd, 1999. 